\title{
Fair social decision under uncertainty and belief disagreements
}

\author{
Takashi Hayashi $^{1} \cdot$ Michele Lombardi $^{1}$
}

Received: 27 April 2017 / Accepted: 22 December 2017 / Published online: 13 January 2018

(C) The Author(s) 2018. This article is an open access publication

\begin{abstract}
This paper aims to address two issues related to simultaneous aggregation of utilities and beliefs. The first one is related to how to integrate both inequality and uncertainty considerations into social decision making. The second one is related to how social decision should take disagreements in beliefs into account. To accomplish this, whereas individuals are assumed to abide by Savage model's of subjective expected utility, society is assumed to prescribe, either to each individual when the ex ante individual well-being is favored or to itself when the ex post individual wellbeing is favored, acting in accordance with the maximin expected utility theory of Gilboa and Schmeidler (J Math Econ 18:141-153, 1989). Furthermore, it adapts an ex ante Pareto-type condition proposed by Gayer et al. (J Legal Stud 43:151-171, 2014), which says that a prospect Pareto dominates another one if the former gives a higher expected utility than the latter one, for each individual, for all individuals' beliefs. In the context where the ex ante individual welfare is favored, our ex ante Pareto-type condition is shown to be equivalent to social utility taking the form of a MaxMinMin social welfare function, as well as to the individual set of priors being contained within the range of individual beliefs. However, when the ex post individual welfare is favored, the same Pareto-type condition is shown to be equivalent to social utility
\end{abstract}

Special thanks go to Philippe Mongin, whose comments and suggestions have led to substantial improvements in the paper. We are also grateful to an associate editor and two referees of this journal for their helpful and thoughtful comments and suggestions. We are solely responsible for any remaining deficiencies.

$凶 \quad$ Takashi Hayashi

takashi.hayashi@glasgow.ac.uk

Michele Lombardi

michele.lombardi@glasgow.ac.uk

1 Adam Smith Business School, University of Glasgow, Glasgow G12 8QQ, UK 
taking the form of a MaxMinMin social welfare function, as well as to the social set of priors containing only weighted averages of individual beliefs.

Keywords Social decision · Uncertainty $\cdot$ Belief disagreements · Fairness

JEL Classification D60 · D63 · D70 · D81

\section{Introduction}

\subsection{Background}

The paper addresses two issues related to the assessment of social prospects under uncertainty (that is, when only subjective probability assessments of the source of uncertainty can be formed). The first one is related to how to integrate both inequality and uncertainty considerations into social decision making. The second one is related to how social decision should take disagreements in beliefs into account. The two issues are inseparably related to one another, as inequality aversion and uncertainty aversion are particularly compelling when individuals disagree on beliefs.

In the context of uncertainty, inequality concerns have generated two approaches to the evaluation of social prospects. The ex ante approach first computes individuals' expected utilities and then assesses prospects by applying a certain egalitarian social welfare criterion to the distributions of ex ante utility values that the computation has generated. The ex post approach first assesses the distributions of individual utility values in each state according to a certain egalitarian social welfare criterion and then evaluates prospects by applying a certain decision-theoretic criterion to these data.

Ben-Porath et al. (1997) claim that neither approach is fully satisfactory. By means of examples which we will revisit below, they aptly argue that to take into account both ex ante and ex post inequality considerations, 'inequality and uncertainty need to be analyzed in tandem' (Ben-Porath et al. 1997, p. 195). In other words, inequality aversion and uncertainty aversion of the 'social observer' are two sides of the same coin. ${ }^{1}$

However, it has been shown (Mongin and Pivato 2015; Zuber 2016) that once we impose both approaches to be commutative with each other, there is no possibility of using any other method of social aggregation aside from utilitarianism, which excludes not only any concern of fairness but also any concern of uncertainty. This impossibility result thus forces us to choose between the two approaches if we want a sufficiently sharp and meaningful social welfare criterion.

This motivates us to characterize a class of social welfare criteria for each approach as well as to integrate as many merits of one approach as possible into the other approach.

Belief disagreement is known to be a severe obstacle for social decision making under uncertainty. Mongin $(1995,1998)$ shows that under belief disagreements the social ordering of prospects cannot satisfy both subjective expected utility (SEU)

\footnotetext{
1 By 'social observer' we do not mean a concrete individual but, instead, a pure aggregate or an abstract entity. 'Social observer' and 'society' will be used interchangeably.
} 
theory à la Savage and the ex ante variant of the Pareto principle, which prescribes that unanimous ex ante preferences should be respected. Chambers and Hayashi (2014) show that the source of the impossibility is that the social welfare criterion is state dependent under ex ante Pareto. For example, if Amy believes it will be sunny and Bob believes it will be rainy, then ex ante Pareto prescribes that Amy should receive more resources if it will be sunny, otherwise Bob should receive more, but such welfare weighting is dependent on whether it is sunny or rainy. Since the standard SEU theory assumes state independence of outcome evaluation, in this paper the social observer is not subjected to SEU theory.

Not only does ex ante Pareto lead to an impossibility but it also has a conceptual problem that Mongin (2016) named as 'spurious unanimity'. Individuals may unanimously agree on ranking uncertain prospects because of double-disagreements in beliefs and tastes. An example by Gilboa et al. (2004) captures this: Amy and Bob are deciding whether to duel or not. Amy believes she will win and she will be happy if she wins. Bob believes he will win and he will be happy if he wins. The ex ante Pareto prescribes that they should duel, but this is an absurd conclusion, as one of them is eventually wrong. ${ }^{2}$

To avoid the problem, Gilboa et al. (2004) proposed an axiom called Consensus Pareto, stating that the ex ante Pareto argument should follow only when all individuals agree on probabilities of relevant events. However, the efficacy of this Pareto axiom to avoid the problem relies on the fact that is formulated in the setting of a rich state space à la Savage. Indeed, in this setup there is a rich class of events such that all individuals agree on their probabilities. However, this is not the case when the state space is not rich, such as when it is finite.

In the context of financial markets, Gayer et al. (2014) have proposed a weaker variant of the standard ex ante Pareto criterion, named Unanimity-Pareto (UP): Prospect $u$ UP-dominates prospect $v$ if $u$ gives a higher expected utility than $v$, for each individual, for all individuals' beliefs. This restricted Pareto not only avoids the problem of spurious unanimity but also provides a collective responsibility for individual beliefs. This motivates us to use this Pareto axiom to check whether it is powerful enough to allow us to rank between uncertain prospects in a discerning manner as well as to aggregate utilities and beliefs into a social preference that is able to balance uncertainty considerations against concerns for equality. Any further step away from the standard ex ante Pareto criterion precipitates an 'excess' paternalism, which is arguable.

\subsection{Outline}

In this paper, an uncertain prospect takes the form of a matrix of utility values, with indices of rows and columns representing individuals and states of nature, respectively. Moreover, we consider the domain of state-contingent utility profiles, where utility values are already risk-adjusted and assumed to be comparable across individuals and states. That is, when an uncertain prospect $u=\left[u_{i s}\right]_{i \in \mathcal{I}, s \in \mathcal{S}}$ is given,

\footnotetext{
2 Although the duel example has recently become popular to illustrate spurious unanimity, Mongin (1997) introduces spurious unanimity in terms of a public good example, which seems to us more plausible.
} 
it means individual $i(\in \mathcal{I})$ receives utility $u_{i s}$ at state $s(\in \mathcal{S})$. Also, we take individual beliefs as parameter inputs. A typical profile of individual beliefs is denoted

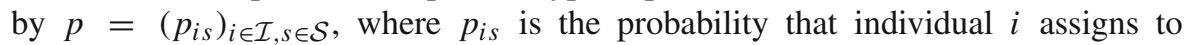
state $s$.

Such specification of the primitives can be justified as follows. When individuals' preferences over bets (random variables) fall in the subjective expected utility theory due to Savage (1972) and Anscombe and Aumann (1963), one can back up their subjective beliefs over states of the world as a unique probability distribution and their von-Neumann Morgenstern utility functions. This does not yet guarantee that such utility functions are interpersonally comparable. For this reason, utility values are taken to be interpersonally comparable throughout the paper.

By endorsing the ex ante egalitarian approach, we obtain that, for a given profile $p$ of individual beliefs, the social observer evaluates uncertain prospects in the form of

$$
\min _{\alpha \in I(p)} \sum_{i \in \mathcal{I}} \alpha_{i}\left(\min _{\sigma_{i} \in S_{i}(p)} \sum_{s \in \mathcal{S}} \sigma_{i s} u_{i s}\right)
$$

where $I(p)$ is the set of welfare weight vectors over individuals which add up to 1 , and $S_{i}(p)$ is the set of beliefs assigned to individual $i$ by the social observer. In other words, he evaluates an uncertain prospect $u$ by first computing its expected value for individual $i$ with respect to the worst belief in $S_{i}(p)$ and then by computing the weighted sum of these data with respect to the vector of welfare weights in $I(p)$ that put greater weight on worse-off individuals.

Here, the size of the set $S_{i}(p)$ reflects how much uncertainty aversion the social observer exercises for individual $i$, whereas the size of $I(p)$ reflects how much the observer is averse to inequality.

However, by favoring the ex post approach, we obtain that for a given profile $p$ uncertain prospects are evaluated by

$$
\min _{\sigma \in S(p)} \sum_{s \in \mathcal{S}} \sigma_{s}\left(\min _{\alpha_{s} \in I_{s}(p)} \sum_{i \in \mathcal{I}} \alpha_{s i} u_{i s}\right)
$$

where $I_{S}(p)$ is a set of state-dependent vectors of welfare weights over individuals which add up to 1 , and $S(p)$ is the social set of beliefs. Thus, to find the evaluation of a prospect, the social observer needs first to compute the worst weighted sum of individuals' utilities in state $s$ by using the weight vectors in $I_{S}(p)$ and then compute the expected value of these data with respect to the worst belief in $S(p)$.

Here, the size of set $I_{S}(p)$ reflects the inequality aversion of the observer in state $s$, whereas the size of set $S(p)$ reflects how much uncertainty aversion the observer exercises for the whole society. Notice that the set $I_{S}(p)$ is state dependent, in general. In this sense, the above representation does not fall into the standard SEU theory which assumes state independence of outcome evaluation. In general, state dependence of an outcome evaluation creates a difficulty in interpreting a subjective belief or set of subjective beliefs, as we cannot distinguish whether the likelihood of some state is 
doubled or the intensity of outcome evaluation at that state is doubled. ${ }^{3}$ However, it is worth mentioning that Karni et al. (1983) and Karni (1985) characterize models of state-dependent preferences. These axiomatizations indicate how additional axioms can permit to identify beliefs in the context of state-dependent preferences.

In the current setting, however, in which we take individual state-contingent utilities to be comparable across individuals and states, it is legitimate to interpret $S(p)$ as the set of social beliefs. This is so because state dependence is only about relative weighting over individuals and, moreover, intensity of evaluation of ex post social welfare is invariant across states. In fact, when everybody receives equal utility at each state, the above representation reduces to the multiple-expected utility that is quite standard in the uncertainty aversion literature.

Further, we also study the consequences of imposing on each class of social decision criteria two Pareto axioms. Despite it being widely recognized that the standard ex ante Pareto principle is problematic, we consider it because our view is that although we should not rely on it, neither should we exclude it altogether. The second one is what we call Collective ex ante Pareto, which is in the spirit of, though logically stronger than, the Unanimity-Pareto proposed by Gayer et al. (2014). More technically, this variant has two parts: (a) Collective ex ante Pareto: if the expected utility of prospect $u$ is at least as large as the expected utility of prospect $v$, for each individual, for all beliefs in $p$, then the social observer should respect this and judge $u$ to be at least as good as $v$; (b) Collective ex ante strict Pareto: if every individual believes that a prospect $u$ is an improvement over another prospect $v$ for herself and for all other individuals and, moreover, she believes that at least one individual becomes strictly better off, then $u$ should be socially preferred to $v$. These properties capture the idea that the society as a whole is responsible for its members' beliefs and, moreover, avoids the spurious unanimity problem.

In the ex ante approach, ex ante Pareto implies that $S_{i}(p)=\left\{p_{i}\right\}$ for each individual $i$, unsurprisingly, meaning that the observer must use individual $i$ 's own belief in order to calculate her ex ante individual welfare, while imposing no restriction on the set of welfare weights. In contrast, Collective ex ante Pareto implies that the set $S_{i}(p)$ of beliefs prescribed by society for individual $i$ lies in the convex hull of individuals' beliefs. This not only allows departure from taking individual belief as it is but also allows for the role of uncertainty aversion. However, the level of uncertainty aversion exercised by society toward each of its members cannot go beyond what is admitted by the range of individuals' beliefs.

In the ex post approach, imposing ex ante Pareto forces us to have only additive aggregation across individuals and states; hence, it leaves no room for inequality aversion and uncertainty aversion. In this sense, the ex post approach is less flexible when

\footnotetext{
3 It is worth mentioning that this problem applies even to the standard SEU theory which assumes state independence of preference. There, after establishing a state-independent preference over outcomes, we establish a state-independent von-Neumann-Morgenstern utility index defined over outcomes. This does not have to be the case, however, because from observed choices alone we can never distinguish between the subjective likelihood of an event and the intensity of the outcome evaluation upon that event. State independence of preference over outcomes does not, by itself, imply state independence of utility scale across states. It is nothing but a modeler's choice to normalize utility scale uniformly across states and to attribute intensity of an outcome evaluation to subjective likelihood of states.
} 
we are more in favor of the spirit of ex ante Pareto. On the other hand, Collective ex ante Pareto allows for the integration of both inequality and uncertainty considerations into social decision making. In this case, the social set of beliefs lies in the convex hull of individuals' beliefs. Thus, society does not add ambiguity beyond a weighted average of individual beliefs.

Finally, based on the prominent examples appearing in the literature, we provide a discussion on which class of social decisions provides more desirable or flexible preference rankings of social prospects.

\subsection{Related literature}

The paper borrows from and extends a number of contributions to the literature on simultaneous aggregation of utilities and beliefs, initiated by Harsanyi's (1955) seminal paper in the context of risk. Harsanyi (1955) shows that when individual and social preferences satisfy the von-Neumann-Morgenstern axioms and, moreover, society abides by ex ante Pareto, then social utility takes the form of a weighted sum of individual utility representations. ${ }^{4}$ However, this social aggregation theorem runs into difficulties when individuals have heterogeneous utilities and beliefs, that is, when we move from risk to uncertainty. Indeed, Mongin (1995) shows that it is impossible to aggregate SEU preferences of individuals into a social ranking that is consistent with SEU theory as well as with ex ante Pareto. Chambers and Hayashi (2014) refine this impossibility result by pinning down the Savage axioms that are incompatible with ex ante Pareto. For a larger class of preferences, Gajdos et al. (2008) show that ex ante Pareto is incompatible with the aggregation of SEU preferences into a consistent social ranking when individuals possess non-neutral attitudes toward uncertainty. ${ }^{5}$

The strategy followed to escape these negative results has been to weaken either $e x$ ante Pareto or the Savage's rationality requirements on either society or individuals, or both, while retaining the ex post variant of the Pareto principle, which prescribes that unanimous ex post preferences should be respected. In this paper, individuals follow the SEU axioms, whereas society is assumed to prescribe, either to each individual when the ex ante individual well-being is favored or to itself when the ex post individual is favored, following the maximin expected utility (MEU) theory of Gilboa and Schmeidler (1989). In contrast to the current literature, the paper offers representation theorems for uncertainty-inequality-sensitive social preferences.

Gilboa et al. (2004) propose a Consensus Pareto axiom according to which the ex ante Pareto argument should be applied only to unambiguous prospects, that is, in those instances in which all individuals agree on probabilities of relevant events. In the framework of Savage (1972), they show that this variant is equivalent to social utility being a weighted average of individuals' utilities as well as to social belief being a

\footnotetext{
${ }^{4}$ Hammond (1981) is an excellent source for the incompatibility problem. By replacing the assumption that the social observer is an expected utility maximizer with the requirement that he follows the state-wise dominance criterion, Fleurbaey (2009) presents two variants of Harsanyi's (1955) utilitarian theorem, one based on continuity and the other on anonymity.

5 Recently, an original form of the impossibility result in which discount rates of individuals play the role of individual priors has been shown by Jackson and Yariv (2015).
} 
weighted average of individuals' beliefs. Furthermore, Alon and Gayer (2016) obtain a generalization of Gilboa et al.'s representation theorem in the case of SEU individuals and an MEU society. They show that a stronger variant of Consensus Pareto implies that the social set of beliefs contains only a weighted average of individuals' beliefs. In the Anscombe and Aumann (1963) framework, Qu (2017) and Danan et al. (2016) derive similar representation theorems for social preferences by employing other variants of ex ante Pareto in a context where neither individuals nor society conforms to SEU theory. ${ }^{6}$ All these variants of ex ante Pareto are proposed to avoid the pitfalls of ex ante Pareto, and especially the problem of 'spurious unanimity', which is at the basis of the impossibility result. In this vein, our Pareto-type condition avoids these problematic cases where individuals agree for opposite reasons by requiring that society should prefer prospect $u$ to prospect $v$ if $u$ constitutes a Pareto improvement over $v$ according to all individuals' beliefs.

As opposed to this and the papers mentioned above, which attempt to distinguish between 'genuine unanimity' and 'spurious unanimity' by relying on individuals' beliefs, Mongin and Pivato (2017) show that this endogenous criterion cannot escape the problem of spurious unanimity when individuals are in a state of complementary ignorance, that is, when individual beliefs arise from conditioning on private information and each individual has information that other members of the society do not have. To escape this problem, they formulate a model which exogenously distinguishes between objective randomness (e.g., risk) and subjective uncertainty. In this setup, they postulate that society abides by what they call objective interim Pareto, according to which the ex ante Pareto argument applies only to cases where individuals agree for objective reasons. They show that if society also abides by ex post Pareto and, moreover, each individual has a well-defined invariant interim preference for each source of uncertainty, then the social and individual preferences have SEU representations. A marked contrast between our result and theirs is that in their framework the Pareto axioms have the feature to endow society with a probability distribution that is not related to individual beliefs.

Billot and Vergopoulos (2014) elaborate with a framework where society is allowed to formulate probability judgements on the opinion each individual has on the actual state of nature, rather than on the actual state as individuals do. They propose a set of Pareto axioms and show that if individuals and society follow the Savage axioms, the Pareto axioms are equivalent to social utility being a convex combination of individual utilities as well as to social belief being an independent product of individual beliefs. This interesting characterization result hinges on the assumption that individuals face independent risk.

Other papers analyze the same issues in the context of financial markets. Posner and Weyl (2013), Blume et al. (2015) and others identify contradictory beliefs as the main cause for purely speculative trades, and they argue in favor of public regulation of financial markets in this case. ${ }^{7}$ Gilboa et al. (2014) formulate a weaker variant of

\footnotetext{
6 Specifically, Qu (2017) assumes that both society and individuals conform to the MEU theory, whereas Danan et al. (2016) consider the possibility that both society and individuals have Bewley-type preferences. For an excellent introduction to the theory of decision under uncertainty, see Gilboa (2009).

7 Blume et al. (2015) propose a Rawlsian-type welfare criterion.
} 
the standard ex ante Pareto criterion, called No-Betting Pareto (NBP), according to which prospect $u$ NBP-dominates prospect $v$ if $u$ Pareto dominates $v$ and if one can find a probability measure $p$ under which each individual strictly prefer $u$ to $v$. They show that NBP-dominance is equivalent to the existence of shared beliefs that can rationalize such preference ranking for each individual and, moreover, they assess the consequences of weakening ex ante Pareto to NBP-dominance comparisons in the context of financial markets. The same type of analysis is continued in Gayer et al. (2014) on the basis of Unanimity-Pareto. Brunnermeier et al. (2014) propose another welfare criterion for markets when individuals hold heterogeneous distorted beliefs. This criterion can be view as a variant of NBP criterion proposed by Gilboa et al. (2014) given that the single belief under which each individual prefers $u$ to $v$ must be a convex combination of individuals' beliefs.

As opposed to the papers mentioned above, which aim to solve the problem of spurious unanimity and to provide utilitarian-type representation theorems for social preferences in various contexts, much less effort has been devoted so far to the consistent aggregation of utilities and beliefs into a social preference that is able to balance uncertainty considerations against a concern for equality.

To comply with Diamond's (1967) thesis that ex ante inequality matters and that it is normatively inappropriate to require that society and individuals abide by the same decision rule, Epstein and Segal (1992) drop the assumption of expected social utility and propose in the context of risk an axiom which captures a social preference for randomization: If society is indifferent between prospect $u$ and prospect $v$, but some individuals strictly prefer $u$ to $v$ and some other individuals strictly prefer $v$ to $u$, then any prospect that is a strictly convex combination of $u$ and $v$ should be strictly preferred to $u$. They also introduce an axiom of mixture symmetry, which prescribes that if society is indifferent between two prospects, then any mixture is socially indifferent to its symmetric counterpart. Under ex ante Pareto and an expected utility requirement on individual preferences, Epstein and Segal derive a quadratic social welfare criterion. Our approach differs from that of Epstein and Segal because for us ex post inequalities also matter.

In an effort to reconcile ex ante and ex post approaches to inequality under uncertainty, Ben-Porath et al. (1997) suggest considering a linear combination of the two approaches, specifically, a linear combination of the expected Gini index and the Gini index of expected income. Indeed, by assuming that society conforms to the MEU theory, they characterize a wide class of min-of-means social welfare functions, which include the Gini social welfare function, minimal income and average income as particular cases. Gajdos and Maurin (2004) extend the idea of Ben-Porath et al. (1997) to any functional that is increasing in both individuals' expected income and equality measurements by making significant additions to Ben-Porath et al.'s (1997) set of axioms. To avoid the impossibility result of Mongin (1995), they impose a weakening of the standard Pareto criterion, which prescribes that society should endorse unanimity when it holds both ex ante and ex post.

The contributions of Ben-Porath et al. (1997) and Gajdos and Maurin (2004) are able to capture both ex ante and ex post equality considerations in the evaluation of income distributions under uncertainty. However, this feature exposes their results to the criticisms that are typically made against each of the approaches, specifically, the 
violation of ex ante Pareto in the ex post approach and the violation of state-wise dominance in the ex ante approach. On this basis, and following the ex post approach proposed by Hammond (1983) and Broome (1991), Fleurbaey (2010) proposes, in the context of risk, a social welfare criterion that behaves as an ex ante criterion in cases where risk generates no inequalities, as an ex post criterion in cases where the final allocation of utilities to individuals is known ex ante; otherwise, the social welfare criterion is a smooth combination of the two approaches to equality. It is worth noting that, though the proposed welfare criterion violates ex ante Pareto, it satisfies both ex ante Pareto and ex ante equality in cases where both prospects are either riskless (that is, when each individual obtains the same units of utility, independent of the state of nature) or egalitarian (that is, when individuals obtain the same units of utility in each realized state of nature). However, since this criterion retains a strong form of consequentialism, it has left open the issue of ex ante equality in lotteries. Fleurbaey (2015) extend Fleurbaey's (2010) analysis to a framework that allows for ex ante equality considerations to play a role in the ex post evaluation. ${ }^{8}$

The remainder of the paper is divided into four sections. Section 2 presents the theoretical framework and outlines the main axioms, whereas the restrictiveness of the weak separability conditions is presented by means of examples in Sect. 3. Section 4 presents our representation theorems, whereas Sect. 5 analyzes the consequences of Collective ex ante Pareto, as well as those of ex ante Pareto, for our classes of social welfare criteria. Sect. 6 concludes.

\section{The theoretical framework and background}

\subsection{The framework}

We denote the set of (mutually exclusive) states of nature by $\mathcal{S}$ and an individual state by $s \in \mathcal{S}$. We assume here that the cardinality of $\mathcal{S}$, denoted by $|\mathcal{S}|$, is finite with $|\mathcal{S}| \geq 2$. As usual, $\Delta(\mathcal{S})$ is the set of probability vectors on $\mathcal{S}$, and $\Delta^{\circ}(\mathcal{S})$ is the set of strictly positive probability vectors. We also assume that individuals belong to a finite set $\mathcal{I}$ with $|\mathcal{I}| \geq 2$ and that individual $i(\in \mathcal{I})$ and society face uncertain prospects. Let $\Delta(\mathcal{I})$ be the set of probability vectors on $\mathcal{I}$, and let $\Delta^{\circ}(\mathcal{I})$ be the set of strictly positive probability vectors. A typical element of $\Delta(\mathcal{I})$ is denoted by $\alpha=\left(\alpha_{i}\right)_{i \in \mathcal{I}}$, where the weight $\alpha_{i}$ can be viewed as the degree of confidence the society has in individual $i$. Throughout the paper, it will be assumed that individuals' risk preferences are fixed and known, whereas individuals' beliefs may vary.

We think of social prospects in the usual way, as functions from states to social consequences, where each social consequence is directly expressed in terms of individuals' utility values, which conceptually amounts to endorsing the questionable thesis of welfarism, that is, that individual utility values summarize all the information required to derive social evaluations. Social prospects are, thus, matrices

\footnotetext{
8 In a model where income distributions are uncertain, Miyagishima (2016) characterizes a maximin social criterion by means of a set of axioms. The value of a prospect is based on the minimum value of individuals' certainty equivalences. A similar result can be found in Fleurbaey and Zuber (2017, proposition 4).
} 
$u=\left[u_{i s}\right]_{i \in \mathcal{I}, s \in \mathcal{S}} \in \mathbb{R}^{|\mathcal{I}| \times|\mathcal{S}|}$, with $u_{i s}$ denoting the utility attained by individual $i$ in state $s$. We write $u_{i}=\left[u_{i s}\right]_{s \in \mathcal{S}} \in \mathbb{R}^{|\mathcal{S}|}$ for the $i$ th row vector of the social prospect $u$, whereas we write $u_{s}=\left[u_{i s}\right]_{i \in \mathcal{I}} \in \mathbb{R}^{|\mathcal{I}|}$ for the $s$ th column vector of $u$. The social prospect $u_{-i}$ is obtained from $u$ by omitting the $i$ th row vector, that is, $u_{-i}=\left(u_{1}, \ldots, u_{i-1}, u_{i+1}, \ldots, u_{|\mathcal{I}|}\right)$, and we identify $\left(u_{i}, u_{-i}\right)$ with $u$. Likewise, $u_{-s}$ is obtained from $u$ by omitting the $s$ th column vector, that is, $u_{-s}$ $=\left(u_{1}, \ldots, u_{s-1}, u_{s+1}, \ldots, u_{|\mathcal{S}|}\right)$, and we identify $\left(u_{s}, u_{-s}\right)$ with $u$. The utility values are assumed to be fully measurable, interpersonally comparable and adjusted for risk. Matrix/vector inequalities are denoted by $\geq,>$ and $\gg . ?$

Society's problem is in how to rank social prospects $u$ in $\mathbb{R}^{|\mathcal{I}| \times|\mathcal{S}|}$. Since individuals' risk preferences are fixed and known, the only information that matters in aggregation is individuals' beliefs. Therefore, given a profile of beliefs $p=\left(p_{i}\right)_{i \in \mathcal{I}} \in(\Delta(\mathcal{S}))^{|\mathcal{I}|}$, the ex ante social preference (at $p$ ) is denoted by $\succsim_{0}^{p} \subseteq \mathbb{R}^{|\mathcal{I}| \times|\mathcal{S}|} \times \mathbb{R}^{|\mathcal{I}| \times|\mathcal{S}|}$. The relations $\succ_{0}^{p}$ and $\sim_{0}^{p}$ are defined as the asymmetric and symmetric parts of $\succsim_{0}^{p}$, as usual. Throughout, we take each $\succsim_{0}^{p}$ to be a continuous weak order (that is, a continuous, complete and transitive binary relation). ${ }^{10}$ The statement $u \succsim_{0}^{p} v$ means that society/social observer judges $u$ to be at least as good as $v$. The statement $u \succ_{0}^{p} v$ means that society judges $u$ better than $v$. Finally, the statement $u \sim^{p} v$ means that society judges $u$ and $v$ as equally good.

\subsection{The theoretical background}

We present some basic axioms for the social preference $\succsim_{0}^{p}$ that will be used in the sequel. For a given profile of beliefs $p$ and for any two social prospects $u$ and $v$ :

\section{A.1. Individual-wise separability ( $\mathcal{I}$-separability, for short):}

For all $i \in \mathcal{I}, \quad\left(u_{i}, u_{-i}\right) \succsim_{0}^{p}\left(v_{i}, u_{-i}\right) \Longleftrightarrow\left(u_{i}, v_{-i}\right) \succsim_{0}^{p}\left(v_{i}, v_{-i}\right)$.

\section{A.2. State-wise separability $(\mathcal{S}$-separability, for short):}

For all $s \in \mathcal{S}, \quad\left(u_{s}, u_{-s}\right) \succsim_{0}^{p}\left(v_{s}, u_{-s}\right) \Longleftrightarrow\left(u_{s}, v_{-s}\right) \succsim_{0}^{p}\left(v_{s}, v_{-s}\right)$.

\section{A.3. Monotonicity:}

(a) $u \geq v \Longrightarrow u \succsim_{0}^{p} v$.

(b) $u>v \Longrightarrow u \succ_{0}^{p} v$.

Throughout the paper, we will say that the social preference $\succsim_{0}$ satisfies an axiom if $\succsim_{0}^{p}$ satisfies that axiom for every $p \in(\Delta(\mathcal{S}))^{|\mathcal{I}|}$.

\footnotetext{
9 We use the following matrix/vector inequalities: for all $u, v \in \mathbb{R}^{|\mathcal{I}| \times|\mathcal{S}|}, u \geq v$ if $u_{i s} \geq y_{i s}$ for all $i$ and all $s ; u>v$ if $u \geq v$ and $u_{i s}>v_{i s}$ for some $i$ and some $s$; and $u \gg v$ if $u_{i s}>y_{i s}$ for all $i$ and all $s$.

10 The social preference $\succsim_{0}^{p}$ is continuous on $\mathbb{R}^{|\mathcal{I}| \times|\mathcal{S}|}$ if for every $u$, the sets $\{v \mid u \succ v\}$ and $\{v \mid v \succ u\}$ are open. Here, we refer to the standard topology on $\mathbb{R}^{|\mathcal{I}| \times|\mathcal{S}|}$. Therefore, the condition is identical to the continuity of consumer preferences in Debreu (1960).
} 
All of the above axioms are quite common. The $\mathcal{I}$-separability axiom and the $\mathcal{S}$-separability axiom are the familiar principles of weak separability. ${ }^{11}$ Then, if $\left(u_{i}, u_{-i}\right) \succsim_{0}^{p}\left(v_{i}, u_{-i}\right)$, this means that individual $i$ 's prospect $u_{i}$ is socially preferred (at $p$ ) to the prospect $v_{i}$ when the full prospect is filled out with $u_{-i}$. When we compare the prospect $\left(u_{i}, v_{-i}\right)$ with $\left(v_{i}, v_{-i}\right)$, we are changing how we fill out the full prospect, but we are still comparing the same two individual $i$ 's prospects. The $\mathcal{I}$-separability axiom, $A .1$, says that the social ranking of $\left(u_{i}, u_{-i}\right)$ and $\left(v_{i}, u_{-i}\right)$ does not change with the filling-out. It says more besides: The same property holds for each individual $i$ in society and each pair of $i$ 's prospects.

The $\mathcal{S}$-separability axiom, $A$.2, instead says that the social ranking between two prospects, $\left(u_{s}, u_{-s}\right)$ and $\left(v_{s}, u_{-s}\right)$, should only depend on the values for which they differ. Indeed, if the state $s$ does not occur, the two prospects result in the exact same allocation $u_{-s}$. Then, when a society compares $\left(u_{s}, u_{-s}\right)$ and $\left(v_{s}, u_{-s}\right)$, it can focus on what happens in state $s$, and ignore the other states.

The monotonicity axiom, A.3, prescribes that society should prefer more to less, no matter at which states the higher payoffs are offered. Note that part $(b)$ prescribes that if $u$ is larger than $v$ for some entries and is no less for any other, then $u$ is better than $v$.

Next, let us define two welfare orderings induced by $\succsim_{0}^{p}$. They are $\succsim_{i}^{p}$ for individual $i$ and $\succsim_{0}^{p, s}$ for society in state $s$. While $\succsim_{i}^{p}$ makes ex ante comparisons, $\succsim_{0}^{p, s}$ makes ex post comparisons. We obtain these orderings by restricting the social preference $\succsim_{0}^{p}$ to prospects that vary along the component of interest.

Definition 1 Suppose that the social preference $\succsim_{0}^{p}$ satisfies the $\mathcal{I}$-separability axiom. For each $i \in \mathcal{I}$, define the following individual $i$ 's ex ante welfare ordering $\succsim_{i}^{p}$ :

$$
u_{i} \succsim_{i}^{p} v_{i} \Longleftrightarrow \text { there are } u, v \in \mathbb{R}^{|\mathcal{I}| \times|\mathcal{S}|} \text { such that } u_{-i}=v_{-i} \text { and } u \succsim_{0}^{p} v \text {. }
$$

Definition 2 Suppose that the social preference $\succsim_{0}^{p}$ satisfies the $\mathcal{S}$-separability axiom. For each $s \in \mathcal{S}$, define the following ex post social welfare ordering in state $s, \succsim_{0}^{p, s}$ :

$$
u_{s} \succsim_{0}^{p, s} v_{s} \Longleftrightarrow \text { there are } u, v \in \mathbb{R}^{|\mathcal{I}| \times|\mathcal{S}|} \text { such that } u_{-s}=v_{-s} \text { and } u \succsim_{0}^{p} v \text {. }
$$

Note that $\succsim_{i}^{p}$ and $\succsim_{0}^{p, s}$ are continuous weak orders; thus, they are representable by continuous real-valued functions. Before stating the consequence of imposing the above weak separability conditions on the social preference, three comments are in order:

Remark 1 One can show that if the social preference satisfies $\mathcal{I}$-separability, then it satisfies the following conditions: (a) Ex ante welfare ordering indifference: if $u_{i}$ and $v_{i}$ are equally good according to individual $i$ 's ex ante welfare ordering and this holds for every individual $i$ (that is, $u_{i} \sim_{i}^{p} v_{i}$ for all $i \in \mathcal{I}$ ), then society should respect this and judge those prospects as equally good (that is, $u \sim_{0}^{p} v$ ); (b) Ex ante welfare

\footnotetext{
11 In multi-attribute decision theory, see Keeney and Raiffa (1993; Ch. 3). In individual decision theory, see Fishburn (1970) and Wakker (1989).
} 
ordering monotonicity: if individual $i$ 's ex ante welfare ordering ranks $u_{i}$ above $v_{i}$ and this holds for every individual $i$ and if for at least one individual $j$ it holds that $u_{j}$ is better than $v_{j}$ (that is, $u_{i} \succsim_{i}^{p} v_{i}$ for all $i \in \mathcal{I}$ and $u_{j} \succ_{j}^{p} v_{j}$ for some $j \in \mathcal{I}$ ), then the society should respect this and judge $u$ better than $v$ (that is, $u \succ_{0}^{p} v$ ).

Remark 2 One can show that $\mathcal{S}$-separability, combined with Definition 2, implies that social preference is increasing with respect to each of its ex post welfare ordering. This is the common state-wise dominance condition, which is considerably weaker than the sure-thing principle-P2 in Savage's system. In individual decision theory, the state-wise dominance condition is regarded as a fundamental tenet for decision making under uncertainty and many non-expected utility models (including the rankdependent utility model) satisfy it.

Remark 3 One can also show that if the social preference satisfies $\mathcal{S}$-separability, then it satisfies the ex post social welfare ordering indifference condition as well as the ex post social welfare ordering monotonicity condition. ${ }^{12}$

The consequence of imposing the $\mathcal{I}$-separability axiom, the $\mathcal{S}$-separability axiom and monotonicity is that the social preferences can be represented, for every profile $p$ of beliefs, in the additive form

$$
u \succsim_{0}^{p} v \Longleftrightarrow \sum_{i \in \mathcal{I}} \sum_{s \in \mathcal{S}} \phi_{i s}\left(u_{i s} \mid p\right) \geq \sum_{i \in \mathcal{I}} \sum_{s \in \mathcal{S}} \phi_{i s}\left(v_{i s} \mid p\right), \quad \text { for all } u, v \in \mathbb{R}^{|\mathcal{I}| \times|\mathcal{S}|}
$$

where $\left\{\phi_{i s}(\cdot \mid p)\right\}_{i \in \mathcal{I}, s \in \mathcal{S}}$ is a family of continuous, increasing and real-valued functions (see Aczel and Maksa 1996; Mongin and Pivato 2015; Zuber 2016). The significance of this representation theorem for us is that the weak separability conditions imply the so-called strong separability condition, which is the most useful necessary and sufficient condition for (social) preferences to be additively separable (see, for instance, Debreu 1960; Fishburn 1970). In other words, we have strong separability when we have weak separability not only for each individual member of the society $\mathcal{I}$ and each individual state of the set $\mathcal{S}$ but also for all subsets formed out of the Cartesian product of those sets. More formally, the strong separability condition states that for any subset $\mathcal{K}$ of the space $\mathcal{I} \times \mathcal{S}$, the social preference is separable on the set $\mathcal{K}$ and on its complement $\mathcal{K}_{C}$.

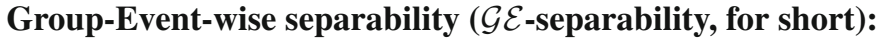

For all $\mathcal{K} \subset \mathcal{I} \times \mathcal{S}, \quad\left(u_{\mathcal{K}}, u_{\mathcal{K}_{C}}\right) \succsim_{0}^{p}\left(v_{\mathcal{K}}, u_{\mathcal{K}_{C}}\right) \Longleftrightarrow\left(u_{\mathcal{K}}, v_{\mathcal{K}_{C}}\right) \succsim_{0}^{p}\left(v_{\mathcal{K}}, v_{\mathcal{K}_{C}}\right)$. 12 Formally, the conditions can be stated as follows: For a given profile of beliefs $p$ and for any two social
prospects $u$ and $v$ :

Ex post social welfare ordering indifference:

$u_{s} \sim_{0}^{p, s} v_{s}$ for all $s \in \mathcal{S} \Longrightarrow u \sim_{0}^{p} v$.

Ex post social welfare ordering monotonicity:

$u_{S} \succsim_{0}^{p, s} v_{S} \quad$ for all $s \in \mathcal{S} \quad$ and $\quad u_{t} \succ_{0}^{p, t} v_{t} \quad$ for some $t \in \mathcal{S} \Longrightarrow u \succ_{0}^{p} v$. 
To be clear, $\mathcal{G} \mathcal{E}$-separability implies the following separability conditions.

\section{Group-wise separability ( $\mathcal{G}$-separability, for short):}

For all $\mathcal{G} \subset \mathcal{I}, \quad\left(u_{\mathcal{G}}, u_{\mathcal{G}_{C}}\right) \succsim_{0}^{p}\left(v_{\mathcal{G}}, u_{\mathcal{G}_{C}}\right) \Longleftrightarrow\left(u_{\mathcal{G}}, v_{\mathcal{G}_{C}}\right) \succsim_{0}^{p}\left(v_{\mathcal{G}}, v_{\mathcal{G}_{C}}\right)$.

\section{Event-wise separability ( $\mathcal{E}$-separability, for short):}

For all $\mathcal{E} \subset \mathcal{S}, \quad\left(u_{\mathcal{E}}, u_{\mathcal{E}_{C}}\right) \succsim_{0}^{p}\left(v_{\mathcal{E}}, u_{\mathcal{E}_{C}}\right) \Longleftrightarrow\left(u_{\mathcal{E}}, v_{\mathcal{E}_{C}}\right) \succsim_{0}^{p}\left(v_{\mathcal{E}}, v_{\mathcal{E}_{C}}\right)$.

\section{The restrictiveness of strong separability}

This section presents, by means of examples, various challenges that the $\mathcal{G} \mathcal{E}$ separability axiom poses in the context of social decisions under uncertainty. ${ }^{13}$

Example 1 First, the $\mathcal{E}$-separability axiom (or the sure-thing principle) is known to be problematic, even in the context of objective risk. In a seminal note, Diamond (1967) argues against the sure-thing principle, contending that, if the society aims to give equal chances to individuals, the social preference should not be subjected to it. The following example illustrates the point.

There are two equally probable states of nature $s_{1}$ and $s_{2}$, and two alternative prospects $u$ and $v$ that a social observer may take. Let us say that the society consists of Amy and Bob, two equally deserving people. Let us then assume that the social observer is symmetric in its treatment of individuals. If prospect $u$ is chosen, there is a probability of 1/2 that Amy will have one unit of utility, while Bob will have none; there is also a probability of $1 / 2$ that Amy and Bob will have zero units of utility. With prospect $v$, it is certain that Amy will have zero units of utility, while there is a probability of 1/2 that Bob will have one unit of utility. In tabular form, we have (Table 1):

An impartial social observer would judge the prospects $u$ and $v$ as equally good (that is, $u \sim_{0} v$ ) because the prospects seem very much the same, except the substitution of the name tags Amy and Bob.

Next, let us consider two alternative prospects, $u^{\prime}$ and $v^{\prime}$, where $u^{\prime}$ (resp., $v^{\prime}$ ) is obtained by replacing the second column of $u$ (resp., $v$ ) with an allocation of utilities that assigns one unit of utility to Amy and none to Bob. In tabular form, we have (Table 2):

Thus, if prospect $u^{\prime}$ is chosen, it is certain that Amy will have one unit of utility, while Bob will have none. With prospect $v^{\prime}$, there is a probability of $1 / 2$ that Amy will have one unit of utility and Bob none, while there is a probability of 1/2 that Amy will have none and Bob will have one unit of utility. According to the $\mathcal{E}$-separability axiom, an impartial social observer needs to judge the new prospects $u^{\prime}$ and $v^{\prime}$ as equally good (that is, $u^{\prime} \sim_{0} v^{\prime}$ ). However, it seems reasonable to judge prospect $v^{\prime}$ better than $u^{\prime}$ since the prospect $u^{\prime}$ discriminates against Bob, while prospect $v^{\prime}$ "gives $\mathrm{B}(\mathrm{ob})$ a fair shake", as Diamond (1967; p. 766) puts it.

\footnotetext{
13 There is a classic stock of arguments for rejecting strong separability in other contexts. See, for instance,
} Gorman (1968). 
Table 1 Example 1, First case

\begin{tabular}{|c|c|c|c|c|c|}
\hline \multicolumn{3}{|c|}{ Prospect $u$} & \multicolumn{3}{|c|}{ Prospect $v$} \\
\hline \multirow[b]{2}{*}{ Agents } & \multicolumn{2}{|c|}{ States of nature } & \multirow[b]{2}{*}{ Agents } & \multicolumn{2}{|c|}{ States of nature } \\
\hline & $s_{1}$ & $s_{2}$ & & $s_{1}$ & $s_{2}$ \\
\hline Amy & 1 & 0 & Amy & 0 & 0 \\
\hline Bob & 0 & 0 & Bob & 1 & 0 \\
\hline
\end{tabular}

Table 2 Example 1, Second case

\begin{tabular}{|c|c|c|c|c|c|}
\hline \multicolumn{3}{|c|}{ Prospect $u^{\prime}$} & \multicolumn{3}{|c|}{ Prospect $v^{\prime}$} \\
\hline \multirow[b]{2}{*}{ Agents } & \multicolumn{2}{|c|}{ States of nature } & \multirow[b]{2}{*}{ Agents } & \multicolumn{2}{|c|}{ States of nature } \\
\hline & $s_{1}$ & $s_{2}$ & & $s_{1}$ & $s_{2}$ \\
\hline Amy & 1 & 1 & Amy & 0 & 1 \\
\hline Bob & 0 & 0 & Bob & 1 & 0 \\
\hline
\end{tabular}

Example 2 As a second example, let us consider again a society composed of two identical individuals, Amy and Bob, facing a choice between two alternative prospects, $u$ and $v$, with two possible and equally probable states of nature, $s_{1}$ and $s_{2}$. Let us also assume that the social observer is symmetric in its treatment of the two individuals. The table below shows what results the prospects would have in the different states of nature (Table 3 ).

An impartial social observer would judge the prospects $u$ and $v$ as equally good because the prospects are very much the same, except the substitution of the name tags Amy and Bob, as well as the name tags state $s_{1}$ and state $s_{2}$. Let us consider now two alternative prospects $u^{\prime}$ and $v^{\prime}$, where $u^{\prime}$ (resp., $v^{\prime}$ ) is derived from prospect $u$ (resp., $v$ ) by assigning one unit of utility to Amy if state $s_{2}$ occurs. In tabular form, we have (Table 4):

If prospect $u^{\prime}$ is chosen, it is certain that Amy will have one unit of utility, while Bob will have none. With prospect $v^{\prime}$, there is a probability of $1 / 2$ that every member of society will have one unit of utility, while there is a probability of $1 / 2$ that everyone will have zero units of utility.

Fleurbaey (2010) proposes two classes of prospects: the riskless prospects (where each individual obtains the same units of utility, independent of the state of nature) and the egalitarian ones (where individuals obtain the same units of utility in each realized state of nature). According to this terminology, $u^{\prime}$ is a riskless prospect while $v^{\prime}$ is an egalitarian one. Indeed, in either state, the utility distribution seems more egalitarian in $v^{\prime}$ than in $u^{\prime}$, and so it seems reasonable on this basis that one ranks $v^{\prime}$ above $u^{\prime}$. However, it is also easy to build an argument in favor of prospect $u^{\prime}$ against prospect $v^{\prime}$ on the basis of "social safety". ${ }^{14}$ Indeed, $u^{\prime}$ is "socially riskless" in the sense that Amy will survive any natural disaster or terrorist attack, whereas $v^{\prime}$ is a "socially risky" prospect, because there is a probability of $1 / 2$ that the entire society will become extinct. We should be able to rank freely the prospects $u^{\prime}$ and $v^{\prime}$, depending

$\overline{14}$ Keeney (1980) and Fishburn (1984) use the notion of "catastrophe avoidance". 
Table 3 Example 2, First case

\begin{tabular}{|c|c|c|c|c|c|}
\hline \multicolumn{3}{|c|}{ Prospect $u$} & \multicolumn{3}{|c|}{ Prospect $v$} \\
\hline \multirow[b]{2}{*}{ Agents } & \multicolumn{2}{|c|}{ States of nature } & \multirow[b]{2}{*}{ Agents } & \multicolumn{2}{|c|}{ States of nature } \\
\hline & $s_{1}$ & $s_{2}$ & & $s_{1}$ & $s_{2}$ \\
\hline Amy & 1 & 0 & Amy & 0 & 0 \\
\hline Bob & 0 & 0 & Bob & 0 & 1 \\
\hline
\end{tabular}

Table 4 Example 2, Second case

\begin{tabular}{|c|c|c|c|c|c|}
\hline \multicolumn{3}{|c|}{ Prospect $u^{\prime}$} & \multicolumn{3}{|c|}{ Prospect $v^{\prime}$} \\
\hline \multirow[b]{2}{*}{ Agents } & \multicolumn{2}{|c|}{ States of nature } & \multirow[b]{2}{*}{ Agents } & \multicolumn{2}{|c|}{ States of nature } \\
\hline & $s_{1}$ & $s_{2}$ & & $s_{1}$ & $s_{2}$ \\
\hline Amy & 1 & 1 & Amy & 0 & 1 \\
\hline Bob & 0 & 0 & Bob & 0 & 1 \\
\hline
\end{tabular}

on which social aspect we believe is more important. But the $\mathcal{G} \mathcal{E}$-separability axiom would make us view the prospects $u^{\prime}$ and $v^{\prime}$ as equally good.

Example 3 As a final example, we show that even the $\mathcal{I}$-separability axiom may be problematic. To illustrate this point, let us consider again a society composed of two identical individuals, Amy and Bob, facing a choice between two alternative prospects, $u$ and $v$, with two possible and equally probable states of nature, $s_{1}$ and $s_{2}$. Let us also assume that the social observer is symmetric in its treatment of the two individuals. The table below shows what results the prospects would have in the different states of nature (Table 5).

An impartial social observer would judge the prospects $u$ and $v$ as equally good because the prospects seem very much the same, except the substitution of the name tags state $s_{1}$ and state $s_{2}$. Also, let us consider two alternative prospects, $u^{\prime}$ and $v^{\prime}$, where $u^{\prime}$ (resp., $v^{\prime}$ ) is derived from prospect $u$ (resp., $v$ ) by assigning one unit of utility to Bob if state $s_{2}$ occurs. In tabular form, we have:

With prospect $u^{\prime}$, there is a probability of $1 / 2$ that Amy will have one unit of utility and Bob none, while there is a probability of $1 / 2$ that Bob will have one unit of utility and Amy none. If prospect $v^{\prime}$ is chosen, there is a probability of $1 / 2$ that every member of society will have one unit of utility, while there is a probability of $1 / 2$ that everyone will have zero units of utility.

In terms of expected utility, both prospects distribute expected utilities equally, and this would make a social observer ex ante indifferent between them-since Amy/Bob has an expected value of $1 / 2$. However, $u^{\prime}$ leads to an unequal ex post distribution of utilities, while $v^{\prime}$ leads to an equal ex post distribution of utilities. Thus, in either state, the utility distribution is more egalitarian in $v^{\prime}$ than in $u^{\prime}$, which may make one rank $v^{\prime}$ above $u^{\prime}$. However, on the basis of "social safety", an opposite conclusion can be supported. Indeed, $u^{\prime}$ is "socially riskless" in the sense that either Amy or Bob will survive any natural disaster, whereas $v^{\prime}$ is a "socially risky" prospect because there is a probability of $1 / 2$ that the entire society will become extinct. Again, we should be able 
Table 5 Example 3, First case

\begin{tabular}{|c|c|c|c|c|c|}
\hline \multicolumn{3}{|c|}{ Prospect $u$} & \multicolumn{3}{|c|}{ Prospect $v$} \\
\hline \multirow[b]{2}{*}{ Agents } & \multicolumn{2}{|c|}{ States of nature } & \multirow[b]{2}{*}{ Agents } & \multicolumn{2}{|c|}{ States of nature } \\
\hline & $s_{1}$ & $s_{2}$ & & $s_{1}$ & $s_{2}$ \\
\hline Amy & 1 & 0 & Amy & 0 & 1 \\
\hline Bob & 0 & 0 & Bob & 0 & 0 \\
\hline
\end{tabular}

Table 6 Example 3, Second case

\begin{tabular}{|c|c|c|c|c|c|}
\hline \multicolumn{3}{|c|}{ Prospect $u^{\prime}$} & \multicolumn{3}{|c|}{ Prospect $v^{\prime}$} \\
\hline \multirow[b]{2}{*}{ Agents } & \multicolumn{2}{|c|}{ States of nature } & \multirow[b]{2}{*}{ Agents } & \multicolumn{2}{|c|}{ States of nature } \\
\hline & $s_{1}$ & $s_{2}$ & & $s_{1}$ & $s_{2}$ \\
\hline Amy & 1 & 0 & Amy & 0 & 1 \\
\hline Bob & 0 & 1 & Bob & 0 & 1 \\
\hline
\end{tabular}

to rank freely the prospects $u^{\prime}$ and $v^{\prime}$, depending on which aspect we believe is more important. But the $\mathcal{I}$-separability axiom would make us view the prospects $u^{\prime}$ and $v^{\prime}$ as equally good and would prevent us from making any social evaluation of the prospects.

\section{Accepting the difference in orders}

The preceding examples suggest that the $\mathcal{I}$-separability axiom and the $\mathcal{S}$-separability axiom are (too) restrictive for a social observer who has, for instance, equality concerns. However, the weak decision-theoretic conditions that lead to the additively separable representation of social preferences give rise to another disturbing result: The compatibility between the ex ante Pareto principle and the ex post Pareto principle can be achieved only if (1) the individuals and the planner follow the SEU axioms (Savage 1972) and (2) they have the same subjective beliefs (Mongin and Pivato 2015).

This is a very stringent conclusion. Indeed, in contrast to classic impossibility results (Harsanyi 1955; Mongin 1995), where it is assumed that individuals and the social observer are expected utility maximizers, we now know that expected utility theory belongs to the conclusions and that the utilitarian-type representation of social preferences basically comes from the weak separability conditions. The classic impossibility results can thus be viewed as a clash between the $\mathcal{I}$-separability axiom and the $\mathcal{S}$-separability axiom. This sounds like a disaster for welfare economics, because these separability conditions are fundamental to (applied) economics.

To escape that negative conclusion, a first rescue move is to explore the consequences of dropping separability altogether. This can be done in a number of conceptual frameworks, given that there are several normatively compelling criteria that one may impose on the individuals and the social observer. ${ }^{15}$ Throughout the rest of the

15 It is already widely known that one way to evade Mongin's (1995) impossibility result is to enlarge the preference domain for society, as shown by Mongin (1998) and Chambers and Hayashi (2006). Specifically, they allow the society to have state-dependent preferences (while remaining in the subjective utility class). 
paper, we posit that individuals follow the SEU axioms (Savage 1972; Anscombe and Aumann 1963). We make this assumption because the key issue here is that society may not satisfy those axioms, even though individuals are SEU maximizers. Moreover, we borrow from decision theory the following well-known axioms: For a given profile of beliefs $p$ and for any two prospects $u$ and $v$ :

\section{A.4. Homogeneity:}

For all $\alpha>0, \quad u \succsim_{0}^{p} v \Longrightarrow \alpha u \succsim_{0}^{p} \alpha v$.

\section{A.5. Certainty-Equality Independence (CE-Independence, for short):}

For all $\alpha \in \mathbb{R}, \quad u \succsim_{0}^{p} v \Longrightarrow u+\alpha \mathbf{1}_{\mathcal{I} \times \mathcal{S}} \succsim_{0}^{p} v+\alpha \mathbf{1}_{\mathcal{I} \times \mathcal{S}}$,

where $\mathbf{1}_{\mathcal{I} \times \mathcal{S}}$ is a matrix of size $|\mathcal{I}|$ by $|\mathcal{S}|$ with 1 's in all its entries.

\section{A.6. Uncertainty-Inequality Aversion (UI-Aversion, for short):}

For all $\alpha \in(0,1), \quad u \sim_{0}^{p} v \Longrightarrow \alpha u+(1-\alpha) v \succsim_{0}^{p} u$.

These are mild but not completely innocuous conditions for social rationality. Let us briefly describe their content. Homogeneity, A.4, is the well-known axiom of invariance of the social ranking under common changes of utility scales. Similarly, CE-Independence, $A .5$, requires the invariance of the social ranking under common changes of utility levels. UI-Aversion, A.6, says that any mixing between two equally good prospects brings social improvement. Thus, it requires that the social observer have a preference for mixing.

Whether one can find the rescue move of dropping separability compelling or not, its well-known consequence within the behavioral tradition of Savage (1972) and Anscombe and Aumann (1963) can be stated as follows:

Lemma 1 Suppose that individuals are SEU maximizers. Suppose that the set of beliefs over $\mathcal{S}$ for individual $i$ is $\Delta^{\circ}(\mathcal{S})$. The continuous weak order $\succsim_{0}$ over $\mathbb{R}^{|\mathcal{I}| \times|\mathcal{S}|}$ satisfies Monotonicity (A.3), Homogeneity ( A.4), CE-Independence (A.5) and UIAversion (A.6) if and only if for every profile of beliefs $p=\left(p_{i}\right)_{i \in \mathcal{I}}$ there exists a unique, closed and convex set of strictly positive probability vectors on $\mathcal{I} \times \mathcal{S}$, $\Lambda(p) \subseteq \Delta^{\circ}(\mathcal{I} \times \mathcal{S})$, such that

$$
\begin{aligned}
u & \gtrsim_{0}^{p} v \Longleftrightarrow \min _{\lambda \in \Lambda(p)}\left(\sum_{i \in \mathcal{I}} \sum_{s \in \mathcal{S}} \lambda_{i s} u_{i s}\right) \\
& \geq \min _{\lambda \in \Lambda(p)}\left(\sum_{i \in \mathcal{I}} \sum_{s \in \mathcal{S}} \lambda_{i s} v_{i s}\right), \quad \text { for all } u, v \in \mathbb{R}^{|\mathcal{I}| \times|\mathcal{S}|} .
\end{aligned}
$$

Proof Let the premises hold. The 'if' part can be checked easily. The 'only if' part follows from Gilboa and Schmeidler (1989; pp. 145-149) by taking the product $\mathcal{I} \times \mathcal{S}$ as the "state space".

Lemma 1 characterizes a criterion for decision making that is quite similar in spirit to that of Gilboa and Schmeidler (1989). Like the MEU model of Gilboa and Schmeidler (1989), the welfare criterion of Lemma 1 enjoys the appeal of simplicity. However, the set $\Lambda(p)$ of beliefs, in comparison, may depend on individuals' beliefs. 
Indeed, in Gilboa and Schmeidler (1989)'s utility model, the decision maker has a subjective set of beliefs and evaluates any prospect by computing its minimum expected value over that set. A distinctive feature of that model is that the set of beliefs is totally subjective. The intuition for this is that when the decision maker has limited or no information about the probability measure that actually governs the phenomenon he observes, the decision maker may consider a range of possible probabilities.

In Lemma 1, the range of probabilities used to compute the minimum possible value of expected utility may be related to the beliefs of individuals. Indeed, the set $\Lambda(p)$ could include, as relevant 'items', probability distributions that are plausible/possible according to individuals' factual or logical knowledge. Of course, the social observer does not commit to those items and use reasons in $\Lambda(p)$ to make his preference judgement. However, since individuals' beliefs can provide the social observer with reasons for his own preference ranking, the above representation theorem may be viewed as an attempt to encompass judgments of how and how much individuals are responsible for their beliefs. Natural candidates for the set $\Lambda(p)$ and their dependence on individuals' beliefs are characterized and discussed in Sect. 5.

Also, the probability distributions in the set $\Lambda(p)$ can be interpreted as social welfare weights. From this viewpoint, the social welfare criterion of Lemma 1 is a weighted sum of individuals' utilities, where for any prospect the weights that give the lowest possible utility sum are used. Thus, this criterion gives the worst-off individuals greater weights than the better-off. Moreover, the larger the size of the set $\Lambda(p)$, the greater the weight placed on worst-off individuals. This produces a range of social welfare criteria that depends on the set $\Lambda(p)$. Since this range encompasses the utilitarian and the Rawlsian criteria as limit points, the social welfare criterion of Lemma 1 can be viewed as a compromise between those "extreme" criteria, and so it may be prima facie considered suitable for making consistent social judgements that address concerns about inequality within the context of uncertainty.

\section{Ex ante form representation theorem}

However, this may be wrong since one can clearly obtain sharper and more meaningful representation theorems for social preferences. Indeed, if at this point one insists on the ex ante individual well-being by making the additional assumption that the social ranking between $\left(u_{i}, u_{-i}\right)$ and $\left(v_{i}, u_{-i}\right)$ should not change with filling-out, he would arrive at the following ex ante form representation theorem of the social preference ranking.

Theorem 1 Under the assumptions of Lemma 1, the continuous weak order $\succsim_{0}$ over $\mathbb{R}^{|\mathcal{I}| \times|\mathcal{S}|}$ satisfies $\mathcal{I}$-separability (A.1) if and only if for every profile of beliefs $p=$ $\left(p_{i}\right)_{i \in \mathcal{I}}$ there exist a unique, closed and convex set of vectors of strictly positive welfare weights on $\mathcal{I}, I(p) \subseteq \Delta^{\circ}(\mathcal{I})$, and a unique family of closed and convex sets of strictly positive probability vectors on $\mathcal{S}, S_{i}(p) \subseteq \Delta^{\circ}(\mathcal{S})$ for each $i \in \mathcal{I}$, such that, for all $u, v \in \mathbb{R}^{|\mathcal{I}| \times|\mathcal{S}|}$, 


$$
\begin{aligned}
& u \succsim_{0}^{p} v \Longleftrightarrow \min _{\alpha \in I(p)} \sum_{i \in \mathcal{I}} \alpha_{i}\left(\min _{\sigma_{i} \in S_{i}(p)} \sum_{s \in \mathcal{S}} \sigma_{i s} u_{i s}\right) \\
& \geq \min _{\alpha \in I(p)} \sum_{i \in \mathcal{I}} \alpha_{i}\left(\min _{\sigma_{i} \in S_{i}(p)} \sum \sigma_{i s} v_{i s}\right) .
\end{aligned}
$$

Proof See Appendix.

In other words, with the addition of the $\mathcal{I}$-separability axiom to Lemma 1 , we can find a set of welfare weights, $I(p)$, as well as a set of probabilities, $S_{i}(p)$, for every individual $i$. To find the evaluation of a prospect $u$, we need first to compute the $e x$ ante individual $i$ 's expected utility of $u$ with respect to the worst probability in the set $S_{i}(p)$, and then compute the minimal weighted average of these data by using the welfare weight vectors in $I(p)$. We interpret the set $I(p)$ of weights as the attitude toward inequality that the social observer may have. By contrast, we view the set $S_{i}(p)$ as the set of recommendations made by the observer to individual $i$ on what set of probabilities individual $i$ needs to base the computation of her own expected utility: Individual $i$ behaves as if any probability distribution that the social observer deems possible is indeed possible.

Note that the observer's recommendations can vary from one individual to another. Therefore, $S_{i}(p)$ reflects the social observer's degree of paternalism and his attitude toward uncertainty. The stronger the degree of paternalism, the less $S_{i}(p)$ may reflect individual $i$ 's own beliefs. One could argue that this is not in the spirit of ex ante solutions, which amount to accepting the individuals' beliefs for better or worse. However, our interpretation of Theorem 1 is as follows: It describes the maximal possible flexibility of recommendations that the oberver can make. Of course, this does not mean that one should be content with any type of recommendation. How the set of recommendations made by the observer to individual $i$ should depend on individual $i$ 's belief is analyzed in Proposition 3 and Theorem 3, where two alternative Pareto-type axioms are considered.

\section{Ex post form representation theorem}

Rather than insisting on the ex ante individual well-being, one could favor the ex post individual well-being by requiring the $\mathcal{S}$-separability axiom. In this case, one would arrive instead at the following ex post form representation theorem for social preference.

Theorem 2 Under the assumptions of Lemma 1, the continuous weak order $\succsim_{0}$ over $\mathbb{R}^{|\mathcal{I}| \times|\mathcal{S}|}$ satisfies $\mathcal{S}$-separability (A.2) if and only if for every profile of beliefs $p=$ $\left(p_{i}\right)_{i \in \mathcal{I}}$ there exist a unique, closed and convex set of strictly positive probability vectors on $\mathcal{S}, S(p) \subseteq \Delta^{\circ}(\mathcal{S})$, and a unique family of closed, convex and statedependent sets of vectors of strictly positive welfare weights on $\mathcal{I}, I_{S}(p) \subseteq \Delta^{\circ}(\mathcal{I})$ for each $s \in \mathcal{S}$, such that, for all $u, v \in \mathbb{R}^{|\mathcal{I}| \times|\mathcal{S}|}$, 


$$
\begin{aligned}
u \succsim_{0}^{p} v \Longleftrightarrow \min _{\sigma \in S(p)} \sum_{s \in \mathcal{S}} \sigma_{s}\left(\min _{\alpha_{s} \in I_{s}(p)} \sum_{i \in \mathcal{I}} \alpha_{s i} u_{i s}\right) \\
\geq \min _{\sigma \in S(p)} \sum_{s \in \mathcal{S}} \sigma_{s}\left(\min _{\alpha_{s} \in I_{S}(p)} \sum \alpha_{s i} v_{i s}\right) .
\end{aligned}
$$

Proof See Appendix.

In other words, with the addition of the $\mathcal{S}$-separability axiom to Lemma 1 , we can find instead a set of state-dependent welfare weights, $I_{S}(p)$, as well as a set of social beliefs, $S(p)$. To find the evaluation of a prospect $u$, we need first to compute the worst weighted sum of individuals' utilities in each state $s$ by using the weight vectors in $I_{S}(p)$ and then compute the expected value of these data with respect to each belief in $S(p)$ and pick the smallest one.

Though the social observer's preference ranking of Theorem 2 violates the eventwise monotonicity principle - that is, P3 in Savage's system-it is legitimate to interpret elements of $S(p)$ as social beliefs. ${ }^{16}$ The reason is that in our framework the cardinal utilities of individuals are fixed, and so the state dependence of the social preference is only about the relative weights of cardinal utilities. This allows us to escape the problem of utility scaling. Note that the larger the size of the set $S(p)$, the greater the degree of uncertainty aversion of the observer. Also, we interpret the state-dependent set $I_{S}(p)$ as the social observer's attitude toward inequality in state $s$.

The above characterization is related to the Multidimensional Generalized Gini criteria axiomatized by Gajdos and Weymark (2005; Theorem 5). Indeed, Gajdos and Weymark (2005) actually provide more general representations because they only use an homogeneity axiom or a translatability axiom (similar to CE-Independence).

\subsection{Comparative inequality/uncertainty aversion}

The remainder of this section is devoted to the concepts of comparative inequality and uncertainty aversion, as well as the consequences for the sets of probability distributions that appear in the above representation theorems.

In the present framework, definitions of comparative inequality aversion are as follows.

Definition 3 For a given profile of beliefs $p$ :

(a) The social preference $\succsim_{0}^{p}$ is more ex ante inequality averse than the social preference $\succsim_{0}^{* p}$ if for all $c \in \mathbb{R}$ and all $u \in \mathbb{R}^{|\mathcal{I}| \times|\mathcal{S}|}$ such that, for each $i \in \mathcal{I}, u_{i s}=u_{i t}$ for all $s, t \in \mathcal{S}$,

$$
c \mathbf{1}_{\mathcal{I} \times \mathcal{S}} \succsim_{0}^{* p} u \Longrightarrow c \mathbf{1}_{\mathcal{I} \times \mathcal{S}} \succsim_{0}^{p} u
$$

\footnotetext{
16 Indeed, when P3 is violated, one is not entitled to consider probability distributions as being subjective probabilities. On this point, see Mongin (1995, 1998) and Chambers and Hayashi (2006).
} 
(b) The ex post welfare ordering $\succsim_{0}^{p, s}$ is more ex post inequality averse than the ex post welfare ordering $\succsim_{0}^{* p, s}$ if for all $c \in \mathbb{R}$ and all $u_{s} \in \mathbb{R}^{|\mathcal{I}|}$,

$$
c \mathbf{1}_{\mathcal{I}} \succsim_{0}^{* p, s} u_{s} \Longrightarrow c \mathbf{1}_{\mathcal{I}} \succsim_{0}^{p, s} u_{s}
$$

where $\mathbf{1}_{\mathcal{I}} \in \mathbb{R}^{|\mathcal{I}|}$ denotes the $|\mathcal{I}|$-dimensional vector whose entries are all 1 and $\mathbf{1}_{\mathcal{I} \times \mathcal{S}} \in \mathbb{R}^{|\mathcal{I}| \times|\mathcal{S}|}$ denotes the $|\mathcal{I}| \times|\mathcal{S}|$-dimensional matrix whose entries are all 1 .

The interpretation of part (a) of this definition is that if a less ex ante inequality averse social observer prefers the egalitarian prospect $c \mathbf{1}_{\mathcal{I}} \times \mathcal{S}$, where all individuals obtain the same unit $c$ of utility across states, to a more unequal distribution of utilities $u$, where utilities can vary across individuals, so does the more ex ante inequality averse social observer. Similarly, the interpretation of part (b) is that if a less ex post inequality averse social observer prefers the egalitarian distribution $c \mathbf{1}_{\mathcal{I}}$ to a more unequal distribution $u_{s}$ so does the more ex post inequality averse social observer.

The next result provides a characterization of the notion of comparative inequality aversion in terms of a simple relation between sets of probability distributions. Let us denote by $I^{*}(p)$ the set of welfare weights induced by the social preference $\succsim_{0}^{* p}$ under the requirements of Theorem 1 , and by $I_{s}^{*}(p)$ the state-dependent set of weight vectors induced by the social preference $\succsim_{0}^{* p}$ under the requirements of Theorem 2 . Therefore:

Proposition 1 For a given profile of beliefs $p$ :

(a) Under the assumptions of Theorem 1, the social preference $\succsim_{0}^{p}$ is more ex ante inequality averse than the social preference $\succsim_{0}^{* p}$ if and only if $I^{*}(p) \subseteq I(p)$.

(b) Under the assumptions of Theorem 2, the ex post welfare ordering $\succsim_{0}^{p, s}$ is more ex post inequality averse at state s than the ex post welfare ordering $\succsim_{0}^{* p, s}$ if and only if $I_{s}^{*}(p) \subseteq I_{s}(p)$.

Proof The statements obviously hold; thus, the proof is omitted.

Comparative uncertainty aversion can be defined as follows.

Definition 4 For a given profile of beliefs $p$ :

(a) The ex ante welfare ordering $\succsim_{i}^{p}$ is more uncertainty averse for individual $i$ than the ex ante welfare ordering $\succsim_{i}^{* p}$ if for all $c \in \mathbb{R}$ and all $u_{i} \in \mathbb{R}^{|\mathcal{S}|}$

$$
c \mathbf{1}_{\mathcal{S}} \succsim_{i}^{* p} u_{i} \Longrightarrow c \mathbf{1}_{\mathcal{S}} \succsim_{i}^{p} u_{i}
$$

(b) The social preference $\succsim_{0}^{p}$ is more socially uncertainty averse than the social preference $\succsim_{0}^{* p}$ if for all $c \in \mathbb{R}$ and all $u \in \mathbb{R}^{|\mathcal{I}| \times|\mathcal{S}|}$ such that, for each $s \in \mathcal{S}, u_{i s}=u_{j s}$ for all $i, j \in \mathcal{I}$,

$$
c \mathbf{1}_{\mathcal{I} \times \mathcal{S}} \succsim_{0}^{* p} u \Longrightarrow c \mathbf{1}_{\mathcal{I} \times \mathcal{S}} \succsim_{0}^{p} u
$$

where $\mathbf{1}_{\mathcal{S}} \in \mathbb{R}^{|\mathcal{S}|}$ denotes the $|\mathcal{S}|$-dimensional vector whose entries are all 1 . 
In other words, part (a) of the definition says that the ex ante welfare ordering $\succsim_{i}^{p}$ is more uncertainty averse than the ex ante welfare ordering $\succsim_{i}^{* p}$, if, whenever $\succsim_{i}^{* p}$ prefers the constant prospect $c \mathbf{1}_{\mathcal{S}}$ to an uncertain prospect $u_{i}$, then the same is true for $\succsim_{i}^{p}$. Similarly, part (b) says that if a less socially uncertainty averse social observer prefers the sure prospect $c \mathbf{1}_{\mathcal{I} \times \mathcal{S}}$ to a more uncertain prospect $u$, where in state $s$ individuals obtain the same level of utility, so does the more socially uncertainty averse social observer.

The next proposition shows that comparative uncertainty attitudes are characterized by a simple relation between sets of probability distributions. To this end, let us denote by $S_{i}^{*}(p)$ the individual $i$ 's set of probability measures induced by the social preference $\succsim_{0}^{* p}$ under the requirements of Theorem 1 , and by $S^{*}(p)$ the set of probability distributions induced by the social preference $\succsim_{0}^{* p}$ under the requirements of Theorem 2. Therefore:

Proposition 2 For a given profile of beliefs $p$ :

(a) Under the assumptions of Theorem 1, the ex ante welfare ordering $\succsim_{i}^{p}$ is more uncertainty averse for individual $i$ than the ex ante welfare ordering $\succsim_{i}^{* p}$ if and only if $S_{i}^{*}(p) \subseteq S_{i}(p)$.

(b) Under the assumptions of Theorem 2, the social preference $\succsim_{0}^{p}$ is more socially uncertainty averse than the social preference $\succsim_{0}^{* p}$ if and only if $S^{*}(p) \subseteq S(p)$.

Proof The statements obviously hold; thus, the proof is omitted.

Although it is clear why $S(p)$ and $S_{i}(p)$ should depend on the profile $p$, their dependence is characterized in the next section. However, one may wonder why $I(p)$ or $I_{S}(p)$ should depend on $p$. In the next section, we show that even if there is no inequality concern, ex ante Pareto implies that ex post welfare weights $I_{S}(p)$ must depend on $p$. For example, if individual $A$ believes that it will be sunny and individual $B$ believe that it will be rainy, then $A$ 's welfare weight will be larger than $B$ 's welfare weight when it is sunny, whereas $B$ 's welfare weight will be larger than $A$ 's welfare weight when it is rainy. Furthermore, under the current scope of the paper, we put no restrictions on the dependence of inequality aversion on belief profile. This is to allow a maximal flexibility of degree of inequality aversion for the society. One may give, however, an informal argument on how much the society should be inequality averse when individuals have more diverse beliefs.

\section{How can each approach deal with disagreements in beliefs?}

\subsection{Ex ante Pareto principle}

In this subsection, we analyze the implications of the ex ante variant of the (strong) Pareto principle for the preceding MaxMinMin representation theorems.

The principle requires that if individuals have the same preference judgment between two prospects, this judgment needs to be compelling for the social observer. More technically, this variant has two parts: (a) Pareto indifference: if every individual judges two social prospects as equally good, then the social observer should respect 
this and judge those prospects as equally good; (b) strict Pareto: if every individual $i$ judges $u_{i}$ to be at least as good as $v_{i}$, and at least one of them judges $u_{j}$ to be better than $v_{j}$, then the social observer should respect this and judge $u$ as better than $v$. In terms of individuals' utilities and their beliefs, the ex ante Pareto principle can be stated as follows: For a given profile of beliefs $p$ and for any two social prospects $u$ and $v$ :

\section{A.7. Ex ante Pareto:}

$$
p_{i} u_{i} \geq p_{i} v_{i} \quad \text { for all } i \in \mathcal{I} \Longrightarrow u \succsim_{0}^{p} v
$$

\section{A.8. Ex ante strict Pareto:}

$$
p_{i} u_{i} \geq p_{i} v_{i} \quad \text { for all } i \in \mathcal{I} \quad \text { and } \quad p_{j} u_{j}>p_{j} v_{j} \quad \text { for some } j \in I \Longrightarrow u \succ_{0}^{p} v \text {. }
$$

The axiom of ex ante Pareto states that if every individual $i$ judges $u_{i}$ to be at least as good as $v_{i}$ according to her own expected utility calculation (that is, $p_{i} u_{i} \geq p_{i} v_{i}$ ), the social observer should judge the social prospect $u$ to be at least as good as $v$. On the other hand, the axiom of ex ante strict Pareto requires that the social observer should judge $u$ to be better than $v$ if every individual $i$ judges $u_{i}$ to be at least as good as $v_{i}$, according to her own expected utility calculation (that is, $p_{i} u_{i} \geq p_{i} v_{i}$ ), and at least one individual $j$ judges $u_{j}$ to be better than $v_{j}$ (that is, $p_{j} u_{j}>p_{j} v_{j}$ ).

A first consequence of the ex ante Pareto principle is that the social observer prefers (weakly) more to less, no matter at which states the higher payoffs are offered. More importantly, it implies that the social observer's ranking of prospects that differs only in what individual $i$ obtains does not change with filling-out.

Lemma 2 Let $p$ be a profile of beliefs. Then, the social preference $\succsim_{0}^{p}$ satisfies Monotonicity and $\mathcal{I}$-separability if it satisfies ex ante Pareto (A.7) as well as ex ante strict Pareto (A.8).

Proof See Appendix.

A second important consequence is that the social observer can no longer take into account ambiguity/uncertainty considerations in his own preference ranking of the prospects. The reason is that the social observer is forced to take individuals' ex ante expected utility calculations as primitives for the evaluation of social prospects. Therefore, and in contrast to the evaluation formula of Theorem 1, the social preference and individuals' preferences are related by a min-of-means formula: The social observer's evaluation of a prospect is the minimal weighted sum, over all weight allocations in $I(p)$, of individuals' ex ante expected utility. This consequence is formalized in the theorem below.

Proposition 3 Under the assumptions of Lemma 1, the continuous weak order $\succsim_{0}$ over $\mathbb{R}^{|\mathcal{I}| \times|\mathcal{S}|}$ satisfies ex ante Pareto (A.7) and ex ante strict Pareto (A.8) if and only if for every profile of beliefs $p=\left(p_{i}\right)_{i \in \mathcal{I}}$ the continuous weak order $\succsim_{0}$ has the ex ante representation form of Theorem 1 where

$$
S_{i}(p)=\left\{p_{i}\right\} \quad \text { for all } i \in \mathcal{I} \text {. }
$$


Proof See Appendix.

Thus, under ex ante Pareto, individual $i$ 's set of recommendations, $S_{i}(p)$, depends uniquely on his belief, meaning that the observer has to accept individual $i$ 's belief for better or worse, and that no correction is required. The consequence is to consider weaker variants of the ex ante Pareto if we want to correct individuals' beliefs. This will be the subject of the next subsection.

When the ex ante Pareto principle is combined with the $\mathcal{S}$-separability axiom, we go back to Harsanyi's Theorem because the social preference over prospects has a weighted utilitarian-type representation, where weights are inferred from considering individuals' own beliefs. This stringent conclusion can be stated as follows.

Proposition 4 Under the assumptions of Lemma 1 , the continuous weak order $\succsim_{0}$ over $\mathbb{R}^{|\mathcal{I}| \times|\mathcal{S}|}$ satisfies $\mathcal{S}$-separability (A.2), ex ante Pareto (A.7) and ex ante strict Pareto (A.8) if and only if there exists a real-valued function $\beta:\left(\Delta^{\circ}(\mathcal{S})\right)^{|\mathcal{I}|} \rightarrow \Delta^{\circ}(\mathcal{I})$ such that for every profile of beliefs $p=\left(p_{i}\right)_{i \in \mathcal{I}}$ :

(a) The continuous weak order $\succsim_{0}^{p}$ has the ex ante representation form of Theorem 1 where

$$
I(p)=\{\beta(p)\} \text {, and } S_{i}(p)=\left\{p_{i}\right\} \quad \text { for all } i \in \mathcal{I} .
$$

(b) The continuous weak order $\succsim_{0}^{p}$ has the ex post representation form of Theorem 2 where

$$
\begin{aligned}
& S(p)=\left\{\left(\sum_{i \in \mathcal{I}} \beta_{i}(p) p_{i s}\right)_{s \in \mathcal{S}}\right\}, \text { and } \\
& I_{s}(p)=\left\{\left(\frac{\beta_{i}(p) p_{i s}}{\sum_{j \in \mathcal{I}} \beta_{j}(p) p_{j s}}\right)_{i \in \mathcal{I}}\right\} \text { for all } s \in \mathcal{S} .
\end{aligned}
$$

Proof See Appendix.

Note that the main culprit for this disappointing result is the ex ante Pareto principle. Also, note that, while Proposition 3 is an ex ante solution that does not include any ex post component, Proposition 4 is an attempt at reconciliation. In this regards, it is worth noting that, similar to ours, the state-dependent solution recently proposed by Mongin and Pivato (2017) also uses individual probabilities to define the ex post weights.

\subsection{Collective ex ante Pareto principle}

Both Proposition 3 and Proposition 4 prove the limitations of the conventional ex ante Pareto principle to social preferences: They exclude any mix between equality and cautiousness from the social observer's eyes. To take into account both inequality considerations and caution concerns, the contribution of the subsection to literature of social decisions under uncertainty is to consider a stronger variant of the UnanimityPareto criterion suggested by Gayer et al. (2014), labeled Collective ex ante Pareto principle, and analyze its implications for our MaxMinMin social judgements. 
To be precise, this variant is based on the idea of unanimity relative to each individual belief: If all individuals agree that prospect $u$ has greater expected utility than prospect $v$ relative to each individual belief, so should the social observer. Formally, it can be stated as follows: For a given profile of beliefs $p$ and for any two prospects $u$ and $v$ :

\section{A.9. Collective ex ante Pareto:}

$$
p_{j} u_{i} \geq p_{j} v_{i} \quad \text { for all } i, j \in \mathcal{I} \Longrightarrow u \succsim_{0}^{p} v .
$$

\section{A.10. Collective ex ante strict Pareto:}

$$
\begin{aligned}
& p_{k} u_{i} \geq p_{k} v_{i} \quad \text { for all } i \in \mathcal{I} \text { and } p_{k} u_{j}>p_{k} v_{j} \quad \text { for some } j \in \mathcal{I}, \\
& \text { for all } k \in \mathcal{I} \Longrightarrow u \succ_{0}^{p} v
\end{aligned}
$$

The axiom of Collective ex ante Pareto states that if the expected utility of prospect $u$ is at least as large as the expected utility of prospect $v$, for each individual and for all beliefs in $p$, then the social observer should respect this and judge $u$ to be at least as good as $v$ (that is, $u \succsim_{0}^{p} v$ ). More intuitively, if no one becomes worse off according to all members' beliefs when we move from $v$ to $u$, then the social observer should admit $u$ as a collective improvement. On the other hand, the axiom of Collective $e x$ ante strict Pareto requires that the social observer should judge $u$ as better than $v$ (that is, $u \succ_{0}^{p} v$ ) if relative to each individual belief, $u$ gives everyone at least as high of expected utility as $v$ and, moreover, it gives at least one individual a strictly higher expected utility than prospect $v$. It should be noted that the social preference satisfies Monotonicity, A.3, if it satisfies the Collective ex ante Pareto principle. Moreover, Collective ex ante strict Pareto implies the Unanimity-Pareto criterion of Gayer et al. (2014), which prescribes that if the expected utility of prospect $u$ is higher than the expected utility of prospect $v$, for each individual, for all beliefs in $p$, then the social observer should judge $u$ to be better than $v$ (that is, $u \succ_{0}^{p} v$ ).

Conceptually, Collective ex ante Pareto principle preserves the individualistic assumption of the classical Pareto principle, which basically means that the social observer is non-creative. His reasons to prefer one prospect to another are based only on the values of individual utilities and their beliefs. Moreover, it assigns a veto power to individuals in situations in which they suffer harm due to other individuals' beliefs. For this reason, it allows us to avoid cases of spurious unanimity (Mongin 2016), in which individuals agree on their preferences over prospects even though they disagree both on beliefs and utilities. Finally, it raises the importance played by individuals' beliefs in providing the observer with reasons for respecting individuals' beliefs even though they are contradictory in situations in which no one is harmed.

Formally, our Collective ex ante Pareto principle is also quite different from the Consensus ex ante Pareto proposed by Gilboa et al. (2004), which states that the Pareto argument should have traction only when individuals agree on probabilities of the relevant events - sets of states. Like our variant, this restriction is able to avoid cases of spurious unanimity. ${ }^{17}$ However, in order for their Consensus Pareto to be

\footnotetext{
17 Though difficulties still remain in situations where individual probabilities arise from conditioning on private information. On this point see Mongin and Pivato (2016, 2017).
} 
powerful enough, it is required that there is a rich class of events for which everybody agrees on their probabilities. In the domain of rich state space, there is a class of such events; this is virtually impossible when the state space is not a continuum, say finite. It is important to emphasize here that Collective ex ante Pareto requires a rich outcome space in order to be powerful.

Given a profile of beliefs $p=\left(p_{i}\right)_{i \in \mathcal{I}}$, let

$$
\mathcal{I}(p)=\left\{J \in 2^{\mathcal{I}} \backslash\{\emptyset\}: p_{i}=p_{j} \quad \text { for all } i, j \in J\right\}
$$

be the partition of the set of individuals such that each element of $\mathcal{I}(p)$ consists of individuals with identical beliefs. To save notation, let $p_{J}$ denote the beliefs commonly held by the members of the set $J \in \mathcal{I}(p)$.

We now show that under the hypotheses and axioms of Lemma 1, the Collective ex ante Pareto principle is equivalent to the existence of a set $\Gamma(p)$ of probability distributions over the set $\mathcal{I} \times \mathcal{I}(p)$ such that the social observer's evaluation of a prospect is the minimal sum of weighted expected utilities. Given any $\gamma \in \Delta^{\circ}(\mathcal{I} \times \mathcal{I}(p))$, $\gamma_{i J}$ explains the weight on individual $i$ 's expected utility that is calculated based on $J$ 's probabilistic opinion. When $i \in J, \gamma_{i J}$ simply explains the weight on $i$ 's expected utility that is calculated based on his belief $i$. However, this calculation is based on other individuals' beliefs when $i \notin J$. Formally:

Lemma 3 Suppose that individuals are SEU maximizers. Suppose that the set of beliefs over $\mathcal{S}$ for individual i is $\Delta^{\circ}(\mathcal{S})$. The continuous weak order $\succsim_{0}$ over $\mathbb{R}^{|\mathcal{I}| \times|\mathcal{S}|}$ satisfies Homogeneity (A.4), CE-Independence (A.5) and UI-Aversion (A.6), and, moreover, it also satisfies Collective ex ante Pareto (A.9) and Collective ex ante strict Pareto (A.10) if and only if for every profile of beliefs $p=\left(p_{i}\right)_{i \in \mathcal{I}}$ there exists a unique, closed and convex set of strictly positive probability vectors on $\mathcal{I} \times \mathcal{I}(p)$, $\Gamma(p) \subseteq \Delta^{\circ}(\mathcal{I} \times \mathcal{I}(p))$, such that, for all $u, v \in \mathbb{R}^{|\mathcal{I}| \times|\mathcal{S}|}$,

$$
\begin{aligned}
& u \succsim_{0}^{p} v \Longleftrightarrow \min _{\gamma \in \Gamma(p)}\left(\sum_{i \in \mathcal{I}} \sum_{J \in \mathcal{I}(p)} \gamma_{i J} \sum_{s \in \mathcal{S}} p_{J s} u_{i s}\right) \\
& \geq \min _{\gamma \in \Gamma(p)}\left(\sum_{i \in \mathcal{I}} \sum_{J \in \mathcal{I}(p)} \gamma_{i J} \sum_{s \in \mathcal{S}} p_{J S} v_{i s}\right) .
\end{aligned}
$$

Proof See Appendix.

It is worth noting that since the profile of beliefs $p$ is in general an $\mathcal{I} \times \mathcal{S}$ matrix with full-rank, under continuity of the social preference with respect to $p$ we can obtain a correspondence $\Gamma:\left(\Delta^{\circ}(\mathcal{S})\right)^{|\mathcal{I}|} \rightarrow 2^{\Delta^{\circ}(\mathcal{I} \times \mathcal{I})}$ by taking limits. Thus, we can resolve the problem of how to count duplicate opinions.

The above representation theorem shows that social preferences of Lemma 1 have substantial flexibility even under the Collective ex ante Pareto principle. The next two results show the consequences of requiring some form of separability of social evaluations. 
On the one hand, whenever one would insist on the ex ante well-being of individuals by requiring the $\mathcal{I}$-separability axiom, the social observer's preference ranking of Lemma 3 takes the form of the evaluation formula of Theorem 1 , where each individual $i$ 's probability distribution over states of the set $S_{i}(p)$ is represented as a profile of weighted means of the non-duplicate beliefs held by the individuals and where each allocation of welfare weights of the set $I(p)$ is represented as a profile of sums where the $i$ th component is represented by the sum of the $i$ th row of the matrix $\gamma \in \Gamma(p)$. These weights reflect social observer's concerns for fairness. Formally, the consequence of positing the $\mathcal{I}$-separability axiom can be stated as follows:

Theorem 3 Under the assumptions of Lemma 3, the continuous weak order $\succsim_{0}$ over $\mathbb{R}^{|\mathcal{I}| \times|\mathcal{S}|}$ satisfies $\mathcal{I}$-separability $(A .1)$ if and only if for every profile of beliefs $p=$ $\left(p_{i}\right)_{i \in \mathcal{I}}$ the continuous weak order $\succsim_{0}$ has the ex ante representation form of Theorem 1 where

$$
I(p)=\left\{\left(\sum_{J \in \mathcal{I}(p)} \gamma_{i J}\right)_{i \in \mathcal{I}}: \gamma \in \Gamma(p)\right\}
$$

and

$$
S_{i}(p)=\left\{\left(\sum_{J \in \mathcal{I}(p)} \gamma_{i J} p_{J S}\right)_{s \in \mathcal{S}}: \gamma \in \Gamma(p)\right\} \quad \text { for all } i \in \mathcal{I} \text {. }
$$

Proof See Appendix.

It is worth emphasizing that according to the above representation theorem the social observer should not impose any extra cautiousness beyond what is admitted by the range of individuals' beliefs. This is a reasonable restriction, although it might be the case that the observer would like to impose extra cautiousness because of paternalistic considerations.

Our sense is that the above theorem is an intermediate case: The observer attributes to each individual $i$ a set of recommendations that are related not only to individual $i$ 's belief, but also to other individuals' beliefs. As monotonicity (A.3) is assumed throughout, the difference between the above theorem and Proposition 3 is purely attributed to the difference in the Pareto-type conditions that are imposed.

On the other hand, one could require that the social observer's preference ranking of Lemma 3 satisfy the $\mathcal{S}$-separability axiom. In this case, his preference ranking would be represented by the evaluation formula of Theorem 2 where each state-dependent vector of weights in $I_{s}(p)$ depends on elements of the set $\Gamma(p)$ and is represented as a normalized weighed average of beliefs held by individuals for state $s$, and where each belief of the social observer also depends on elements of the set $\Gamma(p)$ and is represented as a weighed average of individuals' beliefs. Formally:

Theorem 4 Under the assumptions of Lemma 3, the continuous weak order $\succsim_{0}$ over $\mathbb{R}^{|\mathcal{I}| \times|\mathcal{S}|}$ satisfies $\mathcal{S}$-separability (A.2) if and only if for every profile of beliefs $p=$ $\left(p_{i}\right)_{i \in \mathcal{I}}$ the continuous weak order $\succsim_{0}$ has the ex post representation form of Theorem 2 where 


$$
S(p)=\left\{\left(\sum_{i \in \mathcal{I}} \sum_{J \in \mathcal{I}(p)} \gamma_{i J} p_{J S}\right)_{s \in \mathcal{S}}: \gamma \in \Gamma(p)\right\}
$$

and

$$
I_{S}(p)=\left\{\left(\frac{\sum_{J \in \mathcal{I}(p)} \gamma_{i J} p_{J s}}{\sum_{j \in \mathcal{I}} \sum_{J \in \mathcal{I}(p)} \gamma_{j J} p_{J s}}\right)_{i \in \mathcal{I}}: \gamma \in \Gamma(p)\right\} \quad \text { for all } s \in \mathcal{S}
$$

Proof See Appendix.

The above representation theorem highlights the strength of the $\mathcal{S}$-separability axiom in determining the social observer's aversion to uncertainty, which cannot go beyond what is admitted by the range of individuals' beliefs.

\subsection{Back to the examples}

Going back to the examples presented in Sect. 3, the question that arises is whether our social welfare functions are able to avoid the stringent conclusions drawn from the separability axioms.

In Example 1, we concluded that social indifference between prospects $u^{\prime}$ and $v^{\prime}$ should prevail when the social observer satisfies the axiom of event-wise separability, the states are equiprobable and he judges prospects $u$ and $v$ as equally good. Also, we noted that Diamond (1967) objects to the social indifference between $u^{\prime}$ and $v^{\prime}$ by arguing that in that type of situation one would expect an impartial observer to deem $v^{\prime}$ as better than $u^{\prime}$. The reason for this is that under $v^{\prime}$ Amy and Bob have equal chances to score a high level of utility, whereas $u^{\prime}$ puts Bob at his low level and Amy at her high level, with certainty. On this basis, Diamond (1967) concludes that the social observer should not follow Savages' axioms and calls for social rankings that exhibit more ex ante inequality aversion.

The ex ante social welfare criteria of Proposition 3 and Theorem 3 provide a positive answer to Diamond's call. Indeed, provided that the size of the set of welfare weights, $I(p)$, is large enough, that is, provided that the social observer is sufficiently averse to ex ante inequalities, those social criteria will rank $v^{\prime}$ strictly above $u^{\prime}$. In contrast, the ex post social welfare criteria of Proposition 4 and Theorem 4 judge $u^{\prime}$ and $v^{\prime}$ as equally good. This is so because the axiom of $\mathcal{S}$-separability (A.2) prevents the social observer from endorsing any ex ante inequality aversion.

The objective of Example 2 is to show that an impartial social observer is forced to judge prospect $u^{\prime}$ as equally good as prospect $v^{\prime}$ when he satisfies the group-event-wise separability axiom, the two states are equiprobable and he judges prospects $u$ and $v$ as equally good. We objected against this social indifference by arguing that in that type of situation there are equally compelling reasons for the social observer to judge one prospect better than the other. From an ex ante perspective, one reason for ranking $u^{\prime}$ strictly above $v^{\prime}$ is that $u^{\prime}$ is socially safer than $v^{\prime}$. Indeed, under $u^{\prime}$ Amy will survive any natural disaster while Bob will die certainly, whereas under $v^{\prime}$ Amy and Bob will either both die in the case of a natural disaster or will both survive if no natural disaster 
happens. However, for those who seek an ex post inequality averse criterion, prospect $v^{\prime}$ should be judged to be better than $u^{\prime}$ given that $v^{\prime}$ is less unequal than $u^{\prime}$. Indeed, $v^{\prime}$ puts both Amy and Bob at their high levels in one state and both at their low levels in the other state, whereas $u^{\prime}$ puts Bob at his low level and Amy at her high level, with certainty. Some of the characterized classes partly resolve this objection.

Indeed, on the one hand, the ex ante social welfare criterion of Theorem 3, which assesses prospects by applying the Collective ex ante Pareto principle, induces a strict ranking between $u^{\prime}$ and $v^{\prime}$. More precisely, it would rank $u^{\prime}$ strictly above $v^{\prime}$ when the social observer is not too inequality averse and when every individual $i$ is sufficiently averse to uncertainty. Put differently, the ranking $u^{\prime} \succ_{0}^{p} v^{\prime}$ would prevail when the set $I(p)$ is sufficiently small and the set $S_{i}(p)$ is sufficiently large for each individual $i$. However, the opposite ranking $v^{\prime} \succ_{0}^{p} u^{\prime}$ can be obtained when the social observer is sufficiently averse to inequality as well as when every individual $i$ displays a sufficiently small aversion to uncertainty; that is, the ranking $v^{\prime} \succ_{0}^{p} u^{\prime}$ would prevail when the set $I(p)$ is sufficiently large and the set $S_{i}(p)$ is sufficiently small for each individual $i$.

On the other hand, the ex ante social welfare criterion of Proposition 3, which evaluates prospects on the basis of the ex ante Pareto principle, is much less flexible that the former one. Indeed, for the same reasons seen above, this criterion can induce the ranking $u^{\prime} \succ_{0}^{p} v^{\prime}$. The opposite ranking is still possible when both Amy and Bob believe that state 1 is very likely, but this defense is rather weak and artificial.

Like the ex ante criterion of Theorem 3, the ex post welfare criterion of Theorem 4 is flexible enough to allow the social observer to rank the prospects $u^{\prime}$ and $v^{\prime}$ in either way. Indeed, the ranking $u^{\prime} \succ_{0}^{p} v^{\prime}$ will prevail when $I_{S}(p)$ is not too averse to ex post inequality in each state of nature and the social observer is sufficiently averse to uncertainty, whereas the opposite ranking $v^{\prime} \succ_{0}^{p} u^{\prime}$ will prevail when $I_{s}(p)$ is sufficiently averse to inequality in each state and the social observer exhibits a sufficiently low uncertainty aversion. By contrast, under the ex ante Pareto principle, the welfare criterion of Proposition 4 ranks $u^{\prime}$ and $v^{\prime}$ as equally good. The reason is that the social observer's preference ranking satisfies the group-event-wise separability axiom, that is, $\mathcal{G} \mathcal{E}$-separability.

Example 3 shows that even the $\mathcal{I}$-separability axiom is problematic given that it forces an impartial social observer to view the prospects $u^{\prime}$ and $v^{\prime}$ as equally good, though there are compelling reasons for ranking one prospect strictly above the other. Indeed, one can see from Table 6 that prospect $v^{\prime}$ leads to an ex post equal distribution of utilities, whereas prospect $u^{\prime}$ puts either Amy at her high level and Bob at his low level in state 1, or Amy at her low level and Bob at his high level in state 2. Thus, for those who seek an ex post inequality averse criterion, $v^{\prime}$ should be ranked strictly above $u^{\prime}$ given that $v^{\prime}$ is less unequal than $u^{\prime}$. However, on the basis of social safety, an opposite conclusion can be supported. Indeed, $u^{\prime}$ is socially riskless in the sense that either Amy or Bob will survive any natural disaster, whereas $v^{\prime}$ is a socially risky prospect because there is a probability of $1 / 2$ that the entire society will become extinct. Therefore, this example calls for a flexibility in how the social observer should rank those two prospects.

Unfortunately, our ex ante social welfare criteria of Proposition 3 and Theorem 3 do not leave any room for flexibility because they both satisfy the $\mathcal{I}$-separability 
Table 7 Summary of implications

\begin{tabular}{llll}
\hline Socially desirable ranking & Example 1 & Example 2 & Example 3 \\
\cline { 2 - 3 } & $v^{\prime} \succ_{0}^{p} u^{\prime}$ & Flexible & Flexible \\
\hline Ex ante Pareto: & & & \\
Proposition 3 (ex ante form) & $v^{\prime} \succ_{0}^{p} u^{\prime}$ & $v^{\prime} \succ_{0}^{p} u^{\prime}$ & $v^{\prime} \sim_{0}^{p} u^{\prime}$ \\
Proposition 4 (ex post form) & $v^{\prime} \sim_{0}^{p} u^{\prime}$ & $v^{\prime} \sim_{0}^{p} u^{\prime}$ & $v^{\prime} \sim_{0}^{p} u^{\prime}$ \\
Collective ex ante Pareto: & & & $v^{\prime} \sim_{0}^{p} u^{\prime}$ \\
Theorem 3 (ex ante form) & $v^{\prime} \succ_{0}^{p} u^{\prime}$ & Flexible & Flexible \\
Theorem 4 (ex post form) & $v^{\prime} \sim_{0}^{p} u^{\prime}$ & Flexible & \\
\hline
\end{tabular}

axiom. This negative conclusion is not overturned under the ex ante Pareto principle and the $\mathcal{S}$-separability axiom given that the social preference ranking of Proposition 4 has a weighted utilitarian-type representation. However, the conclusion is overturned by the ex post social welfare criterion of Theorem 4, which evaluates prospects by applying the Collective ex ante Pareto principle. Indeed, on the one hand, this ex post form induces the ranking $u^{\prime} \succ_{0}^{p} v^{\prime}$ when $I_{S}(p)$ is sufficiently ex post utilitarian in each state of nature as well as when the social observer is sufficiently averse to uncertainty. On the other hand, it induces the opposite ranking $v^{\prime} \succ_{0}^{p} u^{\prime}$ when $I_{S}(p)$ is sufficiently averse to inequality in each state of nature as well as when the social observer exhibits a sufficiently low uncertainty aversion.

The above discussion is summarized in Table 7.

\section{Conclusion}

Social preferences of uncertain prospects are representable in the additive form when seemingly mild separability conditions are imposed. This social representation is not fully satisfactory because it excludes not only any concern of fairness but also any concern of uncertainty. Moreover, by common consent, it is desirable to have social welfare rankings of uncertain prospects that take into account both inequality and uncertainty considerations.

On this basis, the paper builds a framework that allows for an explicit incorporation of inequality aversion and uncertainty aversion in the social evaluation of uncertain prospects. It does so by imposing a common set of axioms that capture a notion of social rationality that differs from the standard Bayesian rationality. Indeed, society is assumed to prescribe, either to each individual when the ex ante individual well-being is favored or to itself when the expost individual is favored, following the MEU theory of Gilboa and Schmeidler (1989).

Within this framework, the paper analyzes the consequences of dropping the familiar principles of weak separability, namely, individual-wise separability and state-wise separability. Specifically, by positing individual-wise separability it offers a representation theorem for ex ante uncertainty-inequality-sensitive social preferences. On the other hand, a representation theorem for ex post uncertainty-inequality-sensitive social 
preferences is offered under state-wise separability. The normative appeal of these social representations is that they avoid some of the serious shortcomings implied by the conjunction operation of the separability conditions, addressing particularly the utilitarian indifference to inequalities in utility as shown in the examples of section 3 . Moreover, each representation theorem eases the tension between the two prominent egalitarian approaches to social decision under uncertainty by incorporating as many merits of the other egalitarian approach as possible.

Under individual-wise separability, that is, in the ex ante approach, the social observer assigns a set of beliefs to each individual while he retains for himself a set of welfare weights. On this basis, he evaluates an uncertain prospect by first computing its expected value for each individual $i$ with respect to the worst belief assigned to this individual $i$ and then by computing the weighted sum of these data with respect to the vector of welfare weights that put greater weight on worse-off individuals.

Under state-wise separability, that is, in the ex post approach, the social observer assigns a set of welfare weights to each possible state of nature as well as a set of beliefs to himself. Thus, to find the evaluation of a prospect, the social observer needs first to compute the worst weighted sum of individuals' utilities in each state of nature by using the state-dependent welfare weights and then compute the weighted sum of these data with respect to the worst social belief.

We test how flexible the characterized social preferences are by considering the requirement on how much individuals are responsible for their beliefs.

First, we impose the ordinary ex ante Pareto criterion, which prescribes that if each individual prefers one prospect over another one according to their actual beliefs, then the social observer should respect this. Thus, ex ante Pareto requires that everybody is perfectly and personally responsible for her actual belief. Under ex ante Pareto, the ex ante approach cannot allow any role for an uncertainty aversion, whereas it allows for an inequality aversion freely. On the other hand, the ex post approach excludes not only any concern of uncertainty but also any concern of fairness.

Second, we impose another ex ante Pareto concept that has been suggested elsewhere by Gayer et al. (2014). We name this Pareto criterion as Collective ex ante Pareto. It restricts the standard Pareto argument to cases where each individual believes that a prospect is an improvement over another prospect for herself and for all other individuals. Therefore, it requires a collective evaluation of actual individual beliefs. Under Collective ex ante Pareto, both approaches allow for more desirable and flexible social rankings, while they do not agree in general. Moreover, the social observer cannot exercise any excessive paternalism because the individual set of beliefs and social set of beliefs need to be within what is admitted by the range of individual beliefs.

Let us conclude by discussing future research directions. More realistically, social decisions take place dynamically and so uncertainty resolves gradually as time passes. This creates the problem of how we can make social decisions consistently over time, since there is a tension between ex ante fairness and ex post fairness. This motivates us to investigate how much inequality aversion and ambiguity aversion can be allowed without prejudicing dynamic consistency as well as how such aversions are restricted by how much individuals are responsible for their beliefs. 
Open Access This article is distributed under the terms of the Creative Commons Attribution 4.0 International License (http://creativecommons.org/licenses/by/4.0/), which permits unrestricted use, distribution, and reproduction in any medium, provided you give appropriate credit to the original author(s) and the source, provide a link to the Creative Commons license, and indicate if changes were made.

\section{Appendix}

Proof of Theorem 1 Let the premises hold. It is fairly easy to check that if the social preference has the functional form given in (1), then it satisfies the axioms. Thus, fix any profile of beliefs $p=\left(p_{i}\right)_{i \in \mathcal{I}}$ such that $p_{i} \in \Delta^{\circ}(\mathcal{S})$ and suppose that the continuous weak order $\succsim_{0}^{p}$ satisfies the axioms.

Thus, for each individual $i$, the individual $i$ 's ex ante welfare ordering $\succsim_{i}^{p}$ over $\mathbb{R}^{|\mathcal{S}|}$, given in Definition 1, inherits all properties satisfied by the social preference $\succsim_{0}^{p}$. By Gilboa and Schmeidler (1989), there exists a closed and convex set of strictly positive probability vectors on $\mathcal{S}, S_{i}(p) \subseteq \Delta^{\circ}(\mathcal{S})$, such that the individual $i$ 's ex ante welfare ordering is represented in the form

$$
u_{i} \succsim_{i}^{p} v_{i} \Longleftrightarrow \min _{\sigma_{i} \in S_{i}(p)} \sum_{s \in \mathcal{S}} \sigma_{i s} u_{i s} \geq \min _{\sigma_{i} \in S_{i}(p)} \sum_{s \in \mathcal{S}} \sigma_{i s} v_{i s}, \quad \text { for all } i \in \mathcal{I} \text {. }
$$

Next, given that by Lemma 1 there exists a unique, closed and convex set of strictly positive probability vectors on $\mathcal{I} \times \mathcal{S}, \Lambda(p) \subseteq \Delta^{\circ}(\mathcal{I} \times \mathcal{S})$, let us define the set of welfare weights $I(p)$ by

$$
I(p)=\left\{\left(\sum_{s \in \mathcal{S}} \lambda_{i s}\right)_{i \in \mathcal{I}}: \lambda \in \Lambda(p)\right\} .
$$

It follows from its definition that $I(p)$ is a unique, closed and convex set of strictly positive probability vectors on $\mathcal{I}$.

It suffices to show

$$
\min _{\lambda \in \Lambda(p)} \sum_{i \in \mathcal{I}} \sum_{s \in \mathcal{S}} \lambda_{i s} u_{i s}=\min _{\alpha \in I(p)} \sum_{i \in \mathcal{I}} \alpha_{i}\left(\min _{\sigma_{i} \in S_{i}(p)} \sum_{s \in \mathcal{S}} \sigma_{i s} u_{i s}\right), \quad \text { for all } u \in \mathbb{R}^{|\mathcal{I}| \times|\mathcal{S}|} .
$$

To this end, fix any prospect $u$. For each individual $i \in \mathcal{I}$ and each state $s \in \mathcal{S}$, define $u_{i s}^{*}$ by

$$
u_{i s}^{*}=\min _{\mu_{i} \in S_{i}(p)} \sum_{t \in \mathcal{S}} \mu_{i t} u_{i t} \equiv w_{i}^{*} .
$$

Thus, the matrix $u^{*}=\left[u_{i s}^{*}\right]_{i \in \mathcal{I}, s \in \mathcal{S}} \in \mathbb{R}^{|\mathcal{I}| \times|\mathcal{S}|}$ is a prospect.

By this definition, we have

$$
u_{i}^{*} \sim_{i}^{p} u_{i}, \quad \text { for all } i \in \mathcal{I} \text {, }
$$


where $u_{i}^{*}=\left[u_{i s}^{*}\right]_{s \in \mathcal{S}} \in \mathbb{R}^{|\mathcal{S}|}$ is the $i$ th row vector of the prospect $u^{*}$. Since by remark 1 the social preference satisfies the ex ante welfare ordering indifference condition, it follows that

$$
u^{*} \sim_{0}^{p} u
$$

thus

$$
\min _{\lambda \in \Lambda(p)} \sum_{i \in \mathcal{I}} \sum_{s \in \mathcal{S}} \lambda_{i s} u_{i s}^{*}=\min _{\lambda \in \Lambda(p)} \sum_{i \in \mathcal{I}} \sum_{s \in \mathcal{S}} \lambda_{i s} u_{i s} .
$$

By the definition of the prospect $u^{*}$, we have

$$
\begin{aligned}
\min _{\lambda \in \Lambda(p)} \sum_{i \in \mathcal{I}} \sum_{s \in \mathcal{S}} \lambda_{i s} u_{i s}^{*} & =\min _{\lambda \in \Lambda(p)} \sum_{i \in \mathcal{I}} w_{i}^{*} \sum_{s \in \mathcal{S}} \lambda_{i s} \\
& =\min _{\alpha \in I(p)} \sum_{i \in \mathcal{I}} \alpha_{i} w_{i}^{*} \\
& =\min _{\alpha \in I(p)} \sum_{i \in \mathcal{I}} \alpha_{i}\left(\min _{\sigma_{i} \in S_{i}(p)} \sum_{s \in \mathcal{S}} \sigma_{i s} u_{i s}\right) .
\end{aligned}
$$

Therefore, (5) holds.

Proof of Theorem 2 Though the proof of this representation theorem is similar to that of Theorem 1, we report it for the sake of completeness. Thus, let the premises hold. It is fairly easy to check that if the social preference has the functional form given in (2), then it satisfies the axioms. Thus, fix any profile of beliefs $p=\left(p_{i}\right)_{i \in \mathcal{I}}$ such that $p_{i} \in \Delta^{\circ}(\mathcal{S})$, and suppose that the continuous weak order $\succsim_{0}^{p}$ satisfies the axioms.

Thus, for each state $s$, the ex post welfare ordering $\succsim_{0}^{p, s}$ over $\mathbb{R}^{|\mathcal{I}|}$, given in Definition 2, inherits all properties satisfied by the social preference $\succsim_{0}^{p}$. By Gilboa and Schmeidler (1989), there exists a closed and convex set of strictly positive welfare weights on $\mathcal{I}, I_{s}(p) \subseteq \Delta^{\circ}(\mathcal{I})$, such that the ex post welfare ordering at the state $s$ is represented in the form

$$
u_{s} \succsim_{0}^{p, s} v_{s} \Longleftrightarrow \min _{\alpha_{s} \in I_{s}(p)} \sum_{i \in \mathcal{I}} \alpha_{s i} u_{i s} \geq \min _{\alpha_{s} \in I_{s}(p)} \sum_{i \in \mathcal{I}} \alpha_{s i} v_{i s} .
$$

Next, given that by Lemma 1 there exists a unique, closed and convex set of strictly positive probability vectors on $\mathcal{I} \times \mathcal{S}, \Lambda(p) \subseteq \Delta^{\circ}(\mathcal{I} \times \mathcal{S})$, let us define the set of probability distributions $S(p)$ by

$$
S(p)=\left\{\left(\sum_{i \in \mathcal{I}} \lambda_{i s}\right)_{s \in \mathcal{S}}: \lambda \in \Lambda(p)\right\}
$$

It follows from its definition that $S(p)$ is a unique, closed and convex set of strictly positive probability vectors on $\mathcal{S}$. 
It suffices to show

$$
\min _{\lambda \in \Lambda(p)} \sum_{i \in \mathcal{I}} \sum_{s \in \mathcal{S}} \lambda_{i s} u_{i s}=\min _{\sigma \in S(p)} \sum_{s \in \mathcal{S}} \sigma_{s}\left(\min _{\alpha_{s} \in I_{s}(p)} \sum_{i \in \mathcal{I}} \alpha_{s i} u_{i s}\right), \quad \text { for all } u \in \mathbb{R}^{|\mathcal{I}| \times|\mathcal{S}|} .
$$

Fix any prospect $u$. For each individual $i \in \mathcal{I}$ and each state $s \in \mathcal{S}$, define $u_{i s}^{*}$ by

$$
u_{i s}^{*}=\min _{\alpha_{s} \in I_{s}(p)} \sum_{j \in \mathcal{I}} \alpha_{s j} u_{j s} \equiv w_{s}^{*} .
$$

Thus, the matrix $u^{*}=\left[u_{i s}^{*}\right]_{i \in \mathcal{I}, s \in \mathcal{S}} \in \mathbb{R}^{|\mathcal{I}| \times|\mathcal{S}|}$ is a prospect.

By this definition, we have

$$
u_{s}^{*} \sim_{0}^{p, s} u_{s}, \quad \text { for all } s \in \mathcal{S},
$$

where $u_{s}^{*}=\left[u_{i s}^{*}\right]_{i \in \mathcal{I}} \in \mathbb{R}^{|\mathcal{I}|}$ is the $s$ th column vector of the prospect $u^{*}$. Since by remark 3 the social preference satisfies the ex post welfare ordering indifference condition, it follows that

$$
u^{*} \sim_{0}^{p} u
$$

thus

$$
\min _{\lambda \in \Lambda(p)} \sum_{i \in \mathcal{I}} \sum_{s \in \mathcal{S}} \lambda_{i s} u_{i s}^{*}=\min _{\lambda \in \Lambda(p)} \sum_{i \in \mathcal{I}} \sum_{s \in \mathcal{S}} \lambda_{i s} u_{i s} .
$$

By the definition of the prospect $u^{*}$, we have

$$
\begin{aligned}
\min _{\lambda \in \Lambda(p)} \sum_{i \in \mathcal{I}} \sum_{s \in \mathcal{S}} \lambda_{i s} u_{i s}^{*} & =\min _{\lambda \in \Lambda(p)} \sum_{s \in \mathcal{S}} w_{s}^{*} \sum_{i \in \mathcal{I}} \lambda_{i s} \\
& =\min _{\sigma \in S(p)} \sum_{s \in \mathcal{S}} \sigma_{s} w_{s}^{*} \\
& =\min _{\sigma \in S(p)} \sum_{s \in \mathcal{S}} \sigma_{s}\left(\min _{\alpha_{s} \in I_{s}(p)} \sum_{i \in \mathcal{I}} \alpha_{s i} u_{i s}\right) .
\end{aligned}
$$

Therefore, (6) holds.

Proof of Lemma 2 Fix any profile $p$ of beliefs and any two social prospects $u$ and $v$. Suppose that the social preference $\succsim_{0}^{p}$ satisfies ex ante Pareto and ex ante strict Pareto.

Suppose that $u \geq v$. Then, $u_{i} \geq v_{i}$ for all $i \in \mathcal{I}$. It immediately follows that $p_{i} u_{i} \geq p_{i} v_{i}$ for all $i \in \mathcal{I}$. Then, $u \succsim_{0}^{p} v$ by ex ante Pareto. Hence, the social preference satisfies Monotonicity.

To show that the social preference satisfies $\mathcal{I}$-separability, fix any individual $i$ and suppose that $\left(u_{i}, u_{-i}\right) \succsim_{0}^{p}\left(v_{i}, u_{-i}\right)$. To prove that $\left(u_{i}, v_{-i}\right) \succsim_{0}^{p}\left(v_{i}, v_{-i}\right)$, observe that according to Definition 1 it holds that $u_{i} \succsim_{i}^{p} v_{i}$. Suppose that $p_{i} v_{i}>p_{i} u_{i}$. Then, ex ante strict Pareto implies that $\left(v_{i}, u_{-i}\right) \succ_{0}^{p}\left(u_{i}, u_{-i}\right)$, producing a contradiction. Therefore, we have that $p_{i} u_{i} \geq p_{i} v_{i}$. Since $p_{i} u_{i} \geq p_{i} v_{i}$ and $p_{j} v_{j}=p_{j} v_{j}$ for every 
individual $j \neq i$, Ex ante Pareto implies $\left(u_{i}, v_{-i}\right) \succsim_{0}^{p}\left(v_{i}, v_{-i}\right)$, as we sought. Since the choice of $u_{-i}$ and $v_{-i}$ was arbitrary, we conclude that the social preference satisfies $\mathcal{I}$-separability.

Proof of Proposition 3 Let the premises hold. It is fairly easy to check that if the social preference has the functional form given in (1), then it satisfies the axioms. Thus, fix any profile of beliefs $p=\left(p_{i}\right)_{i \in \mathcal{I}}$ such that $p_{i} \in \Delta^{\circ}(\mathcal{S})$, and suppose that the continuous weak order $\succsim_{0}^{p}$ satisfies the axioms.

Lemma 2 implies that the social preference $\succsim_{0}^{p}$ satisfies Monotonicity and $\mathcal{I}$ separability. Theorem 1 implies that $\succsim_{0}^{p}$ has the ex ante functional form given in (1). What is left is to show that (3) holds.

Fix any individual $i$. First, we show that $p_{i} \in S_{i}(p)$. Assume, to the contrary, that $p_{i} \notin S_{i}(p)$. Thus, the separation theorem implies that there is a nonzero vector $u_{i} \in \mathbb{R}^{|\mathcal{S}|}$ such that

$$
\beta \equiv \min _{\sigma_{i} \in S_{i}(p)} \sigma_{i} u_{i}>p_{i} u_{i}
$$

Fix any real number $\gamma$, and let $\gamma_{-i}$ denote the $|\mathcal{I} \backslash\{i\}| \times|\mathcal{S}|$ matrix with all entries equal to $\gamma$. Moreover, with abuse of notation, we also use $\beta$ to denote the vector in $\mathbb{R}^{|\mathcal{S}|}$ with all entries equal to real number $\beta$. Thus, $\left(u_{i}, \gamma_{-i}\right)$ is a prospect such that $i$ obtains $u_{i}$ and everybody else obtains $\gamma$ with certainty. Also, $\left(\beta, \gamma_{-i}\right)$ is a prospect such that $i$ obtains $\beta$ with certainty and everybody else obtains $\gamma$ with surety.

Since

$$
\min _{\alpha \in I(p)}\left(\alpha_{i} \min _{\sigma_{i} \in S_{i}(p)} \sigma_{i} u_{i}+\gamma \sum_{j \neq i} \alpha_{j}\right)=\min _{\alpha \in I(p)}\left(\alpha_{i} \beta+\gamma \sum_{j \neq i} \alpha_{j}\right),
$$

it follows from (1) that

$$
\left(u_{i}, \gamma_{-i}\right) \succsim_{0}^{p}\left(\beta, \gamma_{-i}\right) .
$$

Furthermore, since $\succsim_{0}^{p}$ satisfies ex ante strict Pareto, it holds that $p_{i} u_{i} \geq p_{i} \beta$. Since $p_{i} \beta=\beta$, we have that $p_{i} u_{i} \geq \beta$, which is a contradiction. Thus, we have that $p_{i} \in S_{i}(p)$.

Next, we show that $S_{i}(p) \subseteq\left\{p_{i}\right\}$. Assume, to the contrary, that $\left\{p_{i}\right\}$ is a proper subset of $S_{i}(p)$. Thus, the separation theorem implies that there is a nonzero vector $u_{i} \in \mathbb{R}^{|\mathcal{S}|}$ such that

$$
p_{i} u_{i}>\min _{\sigma_{i} \in S_{i}(p)} \sigma_{i} u_{i} \equiv \beta .
$$

Again, fix any real number $\gamma$, and let $\gamma_{-i}$ denote the $|\mathcal{I} \backslash\{i\}| \times|\mathcal{S}|$ matrix with all entries equal to $\gamma$. As above, we also use $\beta$ to denote the vector in $\mathbb{R}^{|\mathcal{S}|}$ with all entries equal to real number $\beta$. Thus, since

$$
\min _{\alpha \in I(p)}\left(\alpha_{i} \beta+\gamma \sum_{j \neq i} \alpha_{j}\right)=\min _{\alpha \in I(p)}\left(\alpha_{i} \min _{\mu \in S_{i}(p)} \sigma_{i} u_{i}+\gamma \sum_{j \neq i} \alpha_{j}\right),
$$

we have that

$$
\left(\beta, \gamma_{-i}\right) \succsim_{0}^{p}\left(u_{i}, \gamma_{-i}\right)
$$


Since $\succsim_{0}^{p}$ satisfies ex ante strict Pareto, it holds that $p_{i} \beta \geq p_{i} u_{i}$. Again, since $p_{i} \beta=\beta$, we have that $\beta \geq p_{i} u_{i}$, which is a contradiction. Thus, we conclude that $S_{i}(p)=\left\{p_{i}\right\}$.

Proof of Proposition 4 Let the premises hold. It is fairly easy to check that if the social preference has the functional form given in part (a) or in part (b) of the statement, then it satisfies the axioms. Thus, fix any profile of beliefs $p=\left(p_{i}\right)_{i \in \mathcal{I}}$ such that $p_{i} \in \Delta^{\circ}(\mathcal{S})$, and suppose that the continuous weak order $\succsim_{0}^{p}$ satisfies the axioms.

Since the social preference $\succsim_{0}^{p}$ satisfies ex ante Pareto and ex ante strict Pareto, Lemma 2 implies that it satisfies Monotonicity and $\mathcal{I}$-separability. Thus, the social preference $\succsim_{0}^{p}$ is represented in the additive form by

$$
\sum_{i \in \mathcal{I}} \sum_{s \in \mathcal{S}} \lambda_{i s}(p) u_{i s} \quad \text { for all } u \in \mathbb{R}^{|\mathcal{I}| \times|\mathcal{S}|}
$$

where $\lambda_{i s}(p)>0$ for each individual $i$ and each state $s$ and where $\sum_{i \in \mathcal{I}} \sum_{s \in \mathcal{S}} \lambda_{i s}(p)$ $=1$.

Since by ex ante Pareto the profile $\left(\lambda_{i s}(p)\right)_{s \in \mathcal{S}}$ must be proportional to individual $i$ 's beliefs, $p_{i}, \lambda_{i s}(p)$ has the form

$$
\lambda_{i s}(p)=\beta_{i}(p) p_{i s} \quad \text { for all } s \in \mathcal{S} \text {. }
$$

Observe that $\beta_{i}(p)>0$. Since, moreover,

$$
1=\sum_{i \in \mathcal{I}} \sum_{s \in \mathcal{S}} \lambda_{i s}(p)=\sum_{i \in \mathcal{I}} \sum_{s \in \mathcal{S}} \beta_{i}(p) p_{i s}=\sum_{i \in \mathcal{I}} \beta_{i}(p) \sum_{s \in \mathcal{S}} p_{i s}=\sum_{i \in \mathcal{I}} \beta_{i}(p),
$$

we obtain $\beta(p) \equiv\left(\beta_{i}(p)\right)_{i \in \mathcal{I}} \in \Delta^{\circ}(\mathcal{I})$.

From this, it follows that the social preference $\succsim_{0}^{p}$ is represented in the additive form by

$$
\sum_{i \in \mathcal{I}} \beta_{i}(p) \sum_{s \in \mathcal{S}} p_{i s} u_{i s} \quad \text { for all } u \in \mathbb{R}^{|\mathcal{I}| \times|\mathcal{S}|} .
$$

One can easily see that this corresponds to the ex ante representation form of Theorem 1 with the specification that $I(p)=\{\beta(p)\}$ and $S_{i}(p)=\left\{p_{i}\right\}$ for all $i \in \mathcal{I}$. This completes part (a).

To prove part (b), note that

$$
\begin{aligned}
\sum_{i \in \mathcal{I}} \beta_{i}(p) \sum_{s \in \mathcal{S}} p_{i s} u_{i s} & =\sum_{s \in \mathcal{S}} \sum_{i \in \mathcal{I}} \beta_{i}(p) p_{i s} u_{i s} \\
& =\sum_{s \in \mathcal{S}}\left(\sum_{j \in \mathcal{I}} \beta_{j}(p) p_{j s}\right)\left(\sum_{i \in \mathcal{I}}\left(\frac{\beta_{i}(p) p_{i s}}{\sum_{j \in \mathcal{I}} \beta_{j}(p) p_{j s}}\right) u_{i s}\right) .
\end{aligned}
$$

One can easily check that $\left(\sum_{j \in \mathcal{I}} \beta_{j}(p) p_{j s}\right)_{s \in \mathcal{S}} \in \Delta^{\circ}(\mathcal{S})$ and that $\left(\frac{\beta_{i}(p) p_{i s}}{\sum_{j \in \mathcal{I}} \beta_{j}(p) p_{j s}}\right)_{i \in \mathcal{I}}$ $\in \Delta^{\circ}(\mathcal{I})$ for all $s \in \mathcal{S}$. Therefore, part (b) corresponds to the ex post representation 
form of Theorem 2 where

$$
\begin{aligned}
& S(p)=\left\{\left(\sum_{j \in \mathcal{I}} \beta_{j}(p) p_{j s}\right)_{s \in \mathcal{S}}\right\}, \text { and } \\
& I_{S}(p)=\left\{\left(\frac{\beta_{i}(p) p_{i s}}{\sum_{j \in \mathcal{I}} \beta_{j}(p) p_{j s}}\right)_{i \in \mathcal{I}}\right\} \text { for all } s \in \mathcal{S},
\end{aligned}
$$

as we sought.

Proof of Lemma 3 Let the premises hold. It is fairly easy to check that if the social preference has the functional form given in (4), then it satisfies the axioms. Thus, fix any profile of beliefs $p=\left(p_{i}\right)_{i \in \mathcal{I}}$ such that $p_{i} \in \Delta^{\circ}(\mathcal{S})$, and suppose that the continuous weak order $\succsim_{0}^{p}$ satisfies the axioms.

Let us define the ranking $\succsim_{0}^{* p}$ induced over $\mathbb{R}^{|\mathcal{I}| \times|\mathcal{I}(p)|}$ by the social preference $\succsim_{0}^{p}$ as follows:

$$
u \succsim_{0}^{p} v \Longleftrightarrow x \succsim_{0}^{* p} y, \quad \text { for all } u, v \in \mathbb{R}^{|\mathcal{I}| \times|\mathcal{S}|},
$$

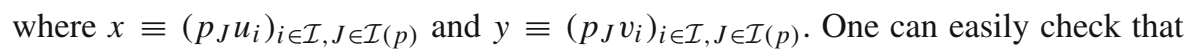
this ranking is complete, transitive, continuous, and convex and, moreover, it satisfies Homogeneity (A.4), UI-Aversion (A.6 ), Collective ex ante Pareto (A.9) and Collective ex ante strict Pareto (A.10).

To show that the ranking also satisfies CE-Independence (A.5), it suffices to show that for all $u, v \in \mathbb{R}^{|\mathcal{I}| \times|\mathcal{S}|}$ it holds

$$
x \succsim_{0}^{* p} y \Longrightarrow x+\alpha \mathbf{1}_{\mathcal{I} \times \mathcal{I}(p)} \succsim_{0}^{* p} y+\alpha \mathbf{1}_{\mathcal{I} \times \mathcal{I}(p)},
$$

where $x \equiv\left(p_{J} u_{i}\right)_{i \in \mathcal{I}, J \in \mathcal{I}(p)}$ and $y \equiv\left(p_{J} v_{i}\right)_{i \in \mathcal{I}, J \in \mathcal{I}(p)}$, where $\mathbf{1}_{\mathcal{I} \times \mathcal{I}(p)} \in \mathbb{R}^{|\mathcal{I}| \times|\mathcal{I}(p)|}$ denotes the $|I| \times|\mathcal{I}(p)|$ matrix whose entries are all 1 and $\alpha$ is a scalar. This follows from the fact that $\succsim_{0}^{p}$ satisfies CE-Independence (A.5) and from the fact that we can take $\left(p_{J}\left(u_{i}+\alpha \mathbf{1}_{\mathcal{S}}\right)\right)_{i \in \mathcal{I}, J \in \mathcal{I}(p)}=x+\alpha \mathbf{1}_{\mathcal{I} \times \mathcal{I}(p)}$ and $\left(p_{J}\left(u_{i}+\alpha \mathbf{1}_{\mathcal{S}}\right)\right)_{i \in \mathcal{I}, J \in \mathcal{I}(p)}=$ $y+\alpha \mathbf{1}_{\mathcal{I} \times \mathcal{I}(p)}$.

Since $\succsim_{0}^{* p}$ satisfies Collective ex ante Pareto (A.9), one can also see that the ranking satisfies Monotonicity (A.3). By Gilboa and Schmeidler (1989), there exists a closed convex set $\Gamma(p) \subseteq \Delta^{\circ}(\mathcal{I} \times \mathcal{I}(p))$ such that, for all $u, v \in \mathbb{R}^{|\mathcal{I}| \times|\mathcal{S}|}$,

$$
x \succsim_{0}^{* p} y \Longleftrightarrow \min _{\gamma \in \Gamma(p)} \sum_{i \in \mathcal{I}} \sum_{J \in \mathcal{I}(p)} \gamma_{i J} x_{i J} \geq \min _{\gamma \in \Gamma(p)} \sum_{i \in \mathcal{I}} \sum_{J \in \mathcal{I}(p)} \gamma_{i J} y_{i J}
$$

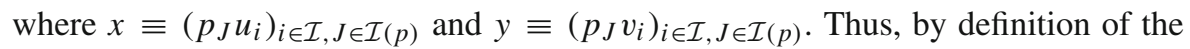
ranking $\succsim_{0}^{* p}$, it follows that for all $u, v \in \mathbb{R}^{|\mathcal{I}| \times|\mathcal{S}|}$ it holds that

$$
u \succsim_{0}^{p} v \Longleftrightarrow \min _{\gamma \in \Gamma(p)} \sum_{i \in I} \sum_{J \in \mathcal{I}(p)} \gamma_{i J} \sum_{s \in \mathcal{S}} p_{J S} u_{i s} \geq \min _{\gamma \in \Gamma(p)} \sum_{i \in I} \sum_{J \in \mathcal{I}(p)} \gamma_{i J} \sum_{s \in \mathcal{S}} p_{J S} u_{i s}
$$

as we sought. 
Proof of Theorem 3 Let the premises hold. It is fairly easy to check that if the social preference has the functional form specified in the statement of Theorem 3 , then it satisfies the axioms. Thus, fix any profile of beliefs $p=\left(p_{i}\right)_{i \in \mathcal{I}}$ such that $p_{i} \in \Delta^{\circ}(\mathcal{S})$, and suppose that the continuous weak order $\succsim_{0}^{p}$ satisfies the axioms.

Thus, for each individual $i$, the individual $i$ 's ex ante welfare ordering $\succsim_{i}^{p}$ over $\mathbb{R}^{|\mathcal{S}|}$, given in Definition 1, inherits all properties satisfied by the social preference $\succsim_{0}^{p}$. By Gilboa and Schmeidler (1989), there exists a closed and convex set of strictly positive probability vectors on $\mathcal{S}, S_{i}(p) \subseteq \Delta^{\circ}(\mathcal{S})$, such that the individual $i$ 's ex ante welfare ordering is represented in the form

$$
u_{i} \succsim_{i}^{p} v_{i} \Longleftrightarrow \min _{\sigma_{i} \in S_{i}(p)} \sum_{s \in \mathcal{S}} \sigma_{i s} u_{i s} \geq \min _{\sigma_{i} \in S_{i}(p)} \sum_{s \in \mathcal{S}} \sigma_{i s} v_{i s}, \quad \text { for all } i \in \mathcal{I} \text {. }
$$

Next, given that by Lemma 3 there exists a unique, closed and convex set of strictly positive probability vectors on $\mathcal{I} \times \mathcal{I}(p), \Gamma(p) \subseteq \Delta^{\circ}(\mathcal{I} \times \mathcal{I}(p))$, let us define the set of welfare weights $I(p)$ by

$$
I(p)=\left\{\left(\sum_{J \in \mathcal{I}(p)} \gamma_{i J}\right)_{i \in \mathcal{I}}: \gamma \in \Gamma(p)\right\}
$$

It follows from its definition that $I(p)$ is a unique, closed and convex set of strictly positive probability vectors on $\mathcal{I}$.

To complete the proof, it suffices to show, for all $u \in \mathbb{R}^{|\mathcal{I}| \times|\mathcal{S}|}$, it holds

$$
\min _{\gamma \in \Gamma(p)}\left(\sum_{i \in \mathcal{I}} \sum_{J \in \mathcal{I}(p)} \gamma_{i J} \sum_{s \in \mathcal{S}} p_{J s} u_{i s}\right)=\min _{\alpha \in I(p)} \sum_{i \in \mathcal{I}} \alpha_{i}\left(\min _{\sigma_{i} \in S_{i}(p)} \sum_{s \in \mathcal{S}} \sigma_{i s} u_{i s}\right) .
$$

To this end, fix any prospect $u$. For each individual $i \in \mathcal{I}$ and each state $s \in \mathcal{S}$, define $u_{i s}^{*}$ by

$$
u_{i s}^{*}=\min _{\mu_{i} \in S_{i}(p)} \sum_{t \in \mathcal{S}} \mu_{i t} u_{i t} \equiv w_{i}^{*} .
$$

Thus, the matrix $u^{*}=\left[u_{i s}^{*}\right]_{i \in \mathcal{I}, s \in \mathcal{S}} \in \mathbb{R}^{|\mathcal{I}| \times|\mathcal{S}|}$ is a prospect.

By this definition, we have

$$
u_{i}^{*} \sim_{i}^{p} u_{i}, \quad \text { for all } i \in \mathcal{I},
$$

where $u_{i}^{*}=\left[u_{i s}^{*}\right]_{s \in \mathcal{S}} \in \mathbb{R}^{|\mathcal{S}|}$ is the $i$ th row vector of the prospect $u^{*}$. Since by remark 1 the social preference satisfies the ex ante welfare ordering indifference condition, it follows that

$$
u^{*} \sim_{0}^{p} u
$$


thus

$$
\min _{\gamma \in \Gamma(p)}\left(\sum_{i \in \mathcal{I}} \sum_{J \in \mathcal{I}(p)} \gamma_{i J} \sum_{s \in \mathcal{S}} p_{J s} u_{i s}^{*}\right)=\min _{\gamma \in \Gamma(p)}\left(\sum_{i \in \mathcal{I}} \sum_{J \in \mathcal{I}(p)} \gamma_{i J} \sum_{s \in \mathcal{S}} p_{J S} u_{i s}\right) .
$$

By the definition of the prospect $u^{*}$, we have

$$
\begin{aligned}
\min _{\gamma \in \Gamma(p)}\left(\sum_{i \in \mathcal{I}} \sum_{J \in \mathcal{I}(p)} \gamma_{i J} \sum_{s \in \mathcal{S}} p_{J s} u_{i s}^{*}\right) & =\min _{\gamma \in \Gamma(p)} \sum_{i \in \mathcal{I}} w_{i}^{*} \sum_{J \in \mathcal{I}(p)} \gamma_{i J} \sum_{s \in \mathcal{S}} p_{J s} \\
& =\min _{\gamma \in \Gamma(p)} \sum_{i \in \mathcal{I}} w_{i}^{*} \sum_{J \in \mathcal{I}(p)} \gamma_{i J} \\
& =\min _{\alpha \in I(p)} \sum_{i \in \mathcal{I}} \alpha_{i} w_{i}^{*} \\
& =\min _{\alpha \in I(p)} \sum_{i \in \mathcal{I}} \alpha_{i}\left(\min _{\sigma_{i} \in S_{i}(p)} \sum_{s \in \mathcal{S}} \sigma_{i s} u_{i s}\right) .
\end{aligned}
$$

Thus, (7) holds. This means that

$$
S_{i}(p)=\left\{\left(\sum_{J \in \mathcal{I}(p)} \gamma_{i J} p_{J S}\right)_{s \in \mathcal{S}}: \gamma \in \Gamma(p)\right\} \quad \text { for all } i \in \mathcal{I},
$$

as we sought.

Proof of Theorem 4 Let the premises hold. It is fairly easy to check that if the social preference has the functional form specified in the statement of Theorem 4 , then it satisfies the axioms. Thus, fix any profile of beliefs $p=\left(p_{i}\right)_{i \in \mathcal{I}}$ such that $p_{i} \in \Delta^{\circ}(\mathcal{S})$, and suppose that the continuous weak order $\succsim_{0}^{p}$ satisfies the axioms.

Thus, for each state $s$, the ex post welfare ordering $\succsim_{0}^{p, s}$ over $\mathbb{R}^{|\mathcal{I}|}$, given in Definition 2, inherits all properties satisfied by the social preference $\succsim_{0}^{p}$. By Gilboa and Schmeidler (1989), there exists a closed and convex set of strictly positive welfare weights on $\mathcal{I}, I_{s}(p) \subseteq \Delta^{\circ}(\mathcal{I})$, such that the ex post welfare ordering at the state $s$ is represented in the form

$$
u_{s} \succsim_{0}^{p, s} v_{s} \Longleftrightarrow \min _{\alpha_{s} \in I_{s}(p)} \sum_{i \in \mathcal{I}} \alpha_{s i} u_{i s} \geq \min _{\alpha_{s} \in I_{s}(p)} \sum_{i \in \mathcal{I}} \alpha_{s i} v_{i s}, \quad \text { for all } s \in \mathcal{S} .
$$

Next, given that by Lemma 3 there exists a unique, closed and convex set of strictly positive probability vectors on $\mathcal{I} \times \mathcal{I}(p), \Gamma(p) \subseteq \Delta^{\circ}(\mathcal{I} \times \mathcal{I}(p))$, let us define the set of social beliefs $S(p)$ by

$$
S(p)=\left\{\left(\sum_{i \in \mathcal{I}} \sum_{J \in \mathcal{I}(p)} \gamma_{i J} p_{J S}\right)_{s \in \mathcal{S}}: \gamma \in \Gamma(p)\right\}
$$


It follows from its definition that $S(p)$ is a unique, closed and convex set of strictly positive probability vectors on $\mathcal{S}$.

To complete the proof, it suffices to show, for all $u \in \mathbb{R}^{|\mathcal{I}| \times|\mathcal{S}|}$, it holds that

$$
\min _{\gamma \in \Gamma(p)} \sum_{i \in \mathcal{I}} \sum_{J \in \mathcal{I}(p)} \gamma_{i J} \sum_{s \in \mathcal{S}} p_{J S} u_{i s}=\min _{\mu \in S(p)} \sum_{s \in \mathcal{S}} \mu_{s} \min _{\alpha_{s} \in I_{s}(p)} \sum_{i \in \mathcal{I}} \alpha_{i s} u_{i s} .
$$

Fix any prospect $u$. For each individual $i \in \mathcal{I}$ and each state $s \in \mathcal{S}$, define $u_{i s}^{*}$ by

$$
u_{i s}^{*}=\min _{\alpha_{s} \in I_{s}(p)} \sum_{j \in \mathcal{I}} \alpha_{s j} u_{j s} \equiv w_{s}^{*} .
$$

Thus, the matrix $u^{*}=\left[u_{i s}^{*}\right]_{i \in \mathcal{I}, s \in \mathcal{S}} \in \mathbb{R}^{|\mathcal{I}| \times|\mathcal{S}|}$ is a prospect.

By this definition, we have

$$
u_{s}^{*} \sim_{0}^{p, s} u_{s}, \quad \text { for all } s \in \mathcal{S},
$$

where $u_{s}^{*}=\left[u_{i s}^{*}\right]_{i \in \mathcal{I}} \in \mathbb{R}^{|\mathcal{I}|}$ is the $s$ th column vector of the prospect $u^{*}$. Since by remark 3 the social preference satisfies the ex post welfare ordering indifference condition, it follows that

$$
u^{*} \sim_{0}^{p} u
$$

thus

$$
\min _{\gamma \in \Gamma(p)} \sum_{i \in \mathcal{I}} \sum_{J \in \mathcal{I}(p)} \gamma_{i J} \sum_{s \in \mathcal{S}} p_{J_{s}} u_{i s}^{*}=\min _{\gamma \in \Gamma(p)} \sum_{i \in \mathcal{I}} \sum_{J \in \mathcal{I}(p)} \gamma_{i J} \sum_{s \in \mathcal{S}} p_{J_{s}} u_{i s} .
$$

By the definition of the prospect $u^{*}$, we also have

$$
\begin{aligned}
\min _{\gamma \in \Gamma(p)} \sum_{i \in \mathcal{I}} \sum_{J \in \mathcal{I}(p)} \gamma_{i J} \sum_{s \in \mathcal{S}} p_{J S} u_{i s}^{*} & =\min _{\gamma \in \Gamma(p)} \sum_{s \in \mathcal{S}}\left(\sum_{J \in \mathcal{I}(p)} \gamma_{i J} \sum_{s \in \mathcal{S}} p_{J S}\right) w_{s}^{*} \\
& =\min _{\sigma \in S(p)} \sum_{s \in \mathcal{S}} \sigma_{s} w_{s}^{*} \\
& =\min _{\sigma \in S(p)} \sum_{s \in \mathcal{S}} \sigma_{s}\left(\min _{\alpha_{s} \in I_{s}(p)} \sum_{i \in \mathcal{I}} \alpha_{s i} u_{i s}\right) .
\end{aligned}
$$

Thus, (8) holds. This means that

$$
I_{S}(p)=\left\{\left(\frac{\sum_{J \in \mathcal{I}(p)} \gamma_{i J} p_{J S}}{\sum_{j \in I} \sum_{J \in \mathcal{I}(p)} \gamma_{j J} p_{J S}}\right)_{i \in \mathcal{I}}: \gamma \in \Gamma(p)\right\} \quad \text { for all } s \in \mathcal{S}
$$

as we sought. 


\section{References}

Alon, S., Gayer, G.: Utilitarian preferences with multiple priors. Econometrica 84, 1181-1201 (2016)

Aczel, J., Maksa, G.: Solution of the rectangular $m \times n$ generalized bisymmetry equation and of the problem of consistent aggregation. J. Math. Anal. Appl. 203, 104-126 (1996)

Anscombe, F.J., Aumann, R.J.: A definition of subjective probability. Ann. Math. Stat. 34, 199-205 (1963)

Ben-Porath, E., Gilboa, I., Schmeidler, D.: On the measurement of Inequality under Uncertainty. J. Econ. Theory 75, 194-204 (1997)

Billot, A., Vergopoulos, V.: Utilitarianism with Prior Heterogeneity (2014). ftp://mse.univ-paris1.fr/pub/ mse/CES2014/14049.pdf

Blume, L.E., Cogley, T., Easley, D.A., Sargent, T.J., Tsyrennikov. V.: A case for incomplete markets (2015). http://www.tomsargent.com/research/case-for-incompleteness.pdf

Broome, J.: Weighing Goods: Equality Uncertainty and Time. Blackwell, Oxford (1991)

Brunnermeier, M.K., Simsek, A., Xiong, W.: A welfare criterion for models with distorted beliefs. Q. J. Econ. 129, 1753-1797 (2014)

Chambers, C.P., Hayashi, T.: Preference aggregation under uncertainty: Savage vs Pareto. Game Econ. Behav. 54, 430-440 (2006)

Chambers, C.P., Hayashi, T.: Preference aggregation with incomplete information. Econometrica 82, 589599 (2014)

Danan, E., Gajdos, T., Hill, B., Tallon, J.-M.: Robust social decisions. Am. Econ. Rev. 106, 2407-2425 (2016)

Debreu, G.: Topological Methods in Cardinal Utility Theory, in Arrow. In: Karlin, S., Suppes, P. (eds.) Mathematical Methods in the Social Sciences 1959, pp. 16-26. Stanford University Press, Palo Alto (1960)

Diamond, P.A.: Cardinal welfare, individualistic ethics, and interpersonal comparison of utility: comment. J. Polit. Econ. 75, 765-766 (1967)

Epstein, L., Segal, U.: Quadratic social welfare functions. J. Polit. Econ. 100, 691-712 (1992)

Fishburn, P.C.: Utility Theory for Decision Making. Pubbl Oper Res Ser 18. Wiley, New York (1970)

Fishburn, P.C.: Equity axioms for public risk. Oper Res 32, 901-908 (1984)

Fleurbaey, M.: Two variants of Harsanyi's aggregation theorem. Econ. Lett. 105, 300-302 (2009)

Fleurbaey, M.: Assessing risky social situations. J. Polit. Econ. 118, 649-680 (2010)

Fleurbaey, M., Gajdos, T., Zuber, S.: Social rationality, separability, and equity under uncertainty. Math. Soc. Sci. 73, 13-22 (2015)

Fleurbaey, M., Zuber, S.: Fair management of social risk. J. Econ. Theory 169, 666-706 (2017)

Gajdos, T., Maurin, E.: Unequal uncertainties and uncertain inequalities: an axiomatic approach. J. Econ. Theory 116, 93-118 (2004)

Gajdos, T., Tallon, J.-M., Vergnaud, J.-C.: Representation and aggregation of preferences under uncertainty. J. Econ. Theory 141, 698-99 (2008)

Gajdos, T., Weymark, J.: Multidimensional generalized Gini indices. Econ. Theory 26, 471-496 (2005)

Gayer, G., Gilboa, I., Samuelson, L., Schmeidler, D.: Pareto efficiency with different beliefs. J. Legal Stud. 43, 151-171 (2014)

Gilboa, I.: Theory of decision under uncertainty. Cambridge University Press, Cambridge (2009)

Gilboa, I., Samuelson, L., Schmeidler, D.: No-betting-Pareto dominance. Econometrica 82, 1405-1442 (2014)

Gilboa, I., Schmeidler, D.: Maxmin expected utility with non-unique priors. J. Math. Econ. 18, 141-153 (1989)

Gilboa, I., Samet, D., Schmeidler, D.: Utilitarian aggregation of beliefs and tastes. J. Polit. Econ. 112, 932-938 (2004)

Gorman, W.: Conditions for additive separability. Econometrica 36, 605-609 (1968)

Hammond, P.J.: Ex ante and ex post welfare optimality under uncertainty. Economics 48, 235-250 (1981)

Hammond, P.J.: Ex post optimality as a dynamically consistent objective for collective choice under uncertainty. In: Pattanaik, P., Salles, M. (eds.) Social Choice and Welfare, pp. 175-205. North-Holland, Amsterdam (1983). Chapter 10

Harsanyi, J.C.: Cardinal welfare, individualistic ethics, and interpersonal comparisons of utility. J. Polit. Econ. 63, 309-321 (1955)

Jackson, M.O., Yariv, L.: Collective dynamic choice: the necessity of time inconsistency. Am Econ J Microecon 7, 150-178 (2015) 
Karni, E., Schmeidler, D., Vind, K.: On state dependent preferences and subjective probabilities. Econometrica 51, 1021-1031 (1983)

Karni, E.: Decision making under uncertainty: the case of state-dependent preferences. Harvard University Press, Cambridge (1985)

Keeney, R.L.: Equity and public risk. Oper. Res. 28, 527-534 (1980)

Keeney, R.L., Raiffa, H.: Decisions with multiple objectives: preferences and value tradeoffs. Wiley, New York (1976). republished by CUP (1993)

Miyagishima, K.: Efficiency, equity, and social rationality under uncertainty (2016) Online https://sites. google.com/site/kanamemiagishimashp/eg

Mongin, P.: Consistent bayesian aggregation. J. Econ. Theory 66, 313-351 (1995)

Mongin, P.: Spurious unanimity and the Pareto principle. THEMA, Universite' de Cergy-Pontoise (working paper, 1997)

Mongin, P.: The Paradox of the Bayesian experts and state-dependent utility theory. J. Math. Econ. 29, 331-361 (1998)

Mongin, P.: Spurious unanimity and the Pareto principle. Econ. Philos. 32(3), 511-532 (2016). https://doi. org/10.1017/S0266267115000371

Mongin, P., Pivato, M.: Ranking multidimensional alternatives and uncertain prospects. J. Econ. Theory 157, 146-171 (2015)

Mongin, P., Pivato, M.: Social evaluation under risk and uncertainty. In: Adler, M., Fleurbaey, M. (eds.) Handbook of Well-Being and Public Policy. OUP, Oxford (2016)

Mongin, P., Pivato, M.: Social preferences under twofold uncertainty, MIMEO (2017)

Posner, E., Weyl, G.: An FDA for Financial Innovation: Applying the Insurable Interest Doctrine to 21st Century Financial Markets, 107 Northwestern University Law Review 1307 (2013)

Qu, X.: Separate aggregation of beliefs and values under ambiguity. Econ. Theory. 63, 503-519 (2017)

Savage, L.J.: The Foundations of Statistics, 2nd edn. Dover, New York (1972)

Wakker, P.P.: Additive Representations of Preferences. Kluwer, Dordrecht (1989)

Zuber, S.: Harsanyi's theorem without the sure-thing principle: on the consistent aggregation of Monotonic Bernoullian and Archimedean preferences. J. Math. Econ. 63, 78-83 (2016) 